\title{
Electrical Characteristics of Quartz-Crystal Units and Their Measurement
}

\author{
By William D. George, Myron C. Selby, and Reuben Scolnik
}

\begin{abstract}
The problem of measuring the dynamic electrical characteristics of high frequency quartz-crystal units was investigated by using ordinary laboratory instruments such as $r-f$ bridges and $Q$ meters. Measurement methods and technics are given, together with relative merits and limitations. Antiresonance impedance up to 5,000,000 ohms was measured to \pm 5 percent or better. Data concerning constancy of electrical characteristics, secondary responses, and changes with amplitude of vibration and temperature are given for a large number of $8.7 M c B T$-cut crystal units having representative types of mountings. Results obtained on a few 50- and 100-kc units are also included. A convenient type of graphical representation of electrical characteristics of normal crystal units is suggested.
\end{abstract}

\section{Introduction}

During the past few years, emergency demands for quartz-crystal units were enormous. Many new methods of production, measurement, and test were intensively studied and used. Precise measurement of the characteristics of each crystal unit was made; however, data concerning the dynamic electric impedance were mostly supplied in an arbitrary manner $[1,2]{ }^{1}$

The problem of measuring a crystal unit as such, i. e., without the use of a particular type of oscillator, was presented by the Army Signal Corps to the Bureau and several other laboratories. The results given in this paper supplement those obtained by others [3, 4] and lead to more exact methods of rating performance and of specifying, measuring, and standardizing those parameters likely to be most useful to the radio and electronics engineer.

A quartz-crystal unit may be precisely specified and measured apart from any external specicuits with which it is used. This has been proved on various occasions in print and in discussion $[3,5,7]$. This paper reviews ways of expressing the equivalent electric circuit and describes measurement methods and technics by which one may determine the electrical characteristics of a crystal unit over a wide range of frequency

1 Figures in brackets indicate the literature references at the end of this paper. and impedance, with such commonly used equipment as $\mathrm{r}-\mathrm{f}$ bridges and $Q$-meters; it shows how one may present the performance characteristics in a simple way to assist in differentiating between a normal unit and one remaining constant only under a limited set of conditions.

The "activity" of a crystal unit is sometimes defined in terms of both grid current and speed with which oscillations start and stop when a test oscillator is keyed. Certain applications of crystal units require data concerning electrical, mechanical, and thermal transients. Such data, including overload or burnout-characteristics, are not dealt with in this paper. However, they may be associated with and supplement any desirable steady state index of performance.

The measurement methods described were useful in exploring nearby or overlapping secondary responses and regions above and below the maximum antiresonance frequency of the crystal unit. Constancy of electrical characteristics from day to day and changes with temperature and amplitude of vibration were investigated for a large number of $8.7-\mathrm{Mc}$, BT-cut units having representative types of mountings. Changes in the fundamental electrical equivalent characteristics were not observed in selected units at crystal currents up to about 100 ma. Data concerning the foregoing are presented in graphical form. Results obtained on a few 50- and 100-kc units, having metal-film electrodes, are also given. 


\section{Equivalent Circuit Representing A Normal Mode of Vibration}

Piezoelectric crystals were introduced into the radio-frequency field to meet the need for a resonant circuit (either series or parallel) having constant parameters and low losses (high Q). A fundamental requirement in this application was therefore that the quartz-crystal unit be the electrical equivalent of a single resonant (or antiresonant) circuit. The unit that most nearly meets this requirement may be referred to as a normal crystal. Other units having interfering responses, or closely spaced multiple resonances, are apparently equivalent to electric-circuit meshes having a number of mutually coupled resonant circuits. These may be treated as complex crystals and should be dealt with separately.

It should be pointed out, however, that this differentiation between "normal" and "complex" crystal units applies to the major mode of vibration of a crystal unit and to the responses that may be immediately next to it.

The equivalent circuit of a normal crystal is shown in figure 1, a. It has one resonant $\left(f_{r}\right)$ and one antiresonant $\left(f_{a}\right)$ frequency. The impedance across its terminals may well be illustrated by means of the susceptance and reactance diagrams shown in figure 1 , c and d. $R_{s}$ is small compared with $\omega L$ and can be neglected in these diagrams.

The susceptance $\left(B_{a b c}\right)$ of the $L_{1}, C_{1}$ series branch is added to the susceptance $\left(B_{t}\right)$ of the shunt capacity $C_{t} . \quad C_{t}$ is the total shunt capacity across the crystal and is usually designated as $C_{0}$ when the external capacity is zero. The resultant $B_{e}$ is obtained in figure 1 , c as the resultant termination reactance $X_{e}$ in figure $1, \mathrm{~d}$. It is evident from the diagram that as $C_{t}$ is increased in value the slope of $B_{t}$ will increase and $f_{a}$ will approach $f_{r}$. It is also apparent that $X_{e}$ is positive between $f_{r}$ and $f_{a}$. The equivalent circuit at frequencies in this range is therefore as shown in figure $1, \mathrm{~b}$, where $R_{e}$ is the resistive component, including the effect of the series resistance $R_{s}$ and all other losses of the unit. When such a circuit is tuned to antiresonance with an external capacitor, the termination impedance is resistive and is equal to

$$
R_{p}=Q_{e} X_{e}=\frac{X_{e}^{2}}{R_{e}}
$$
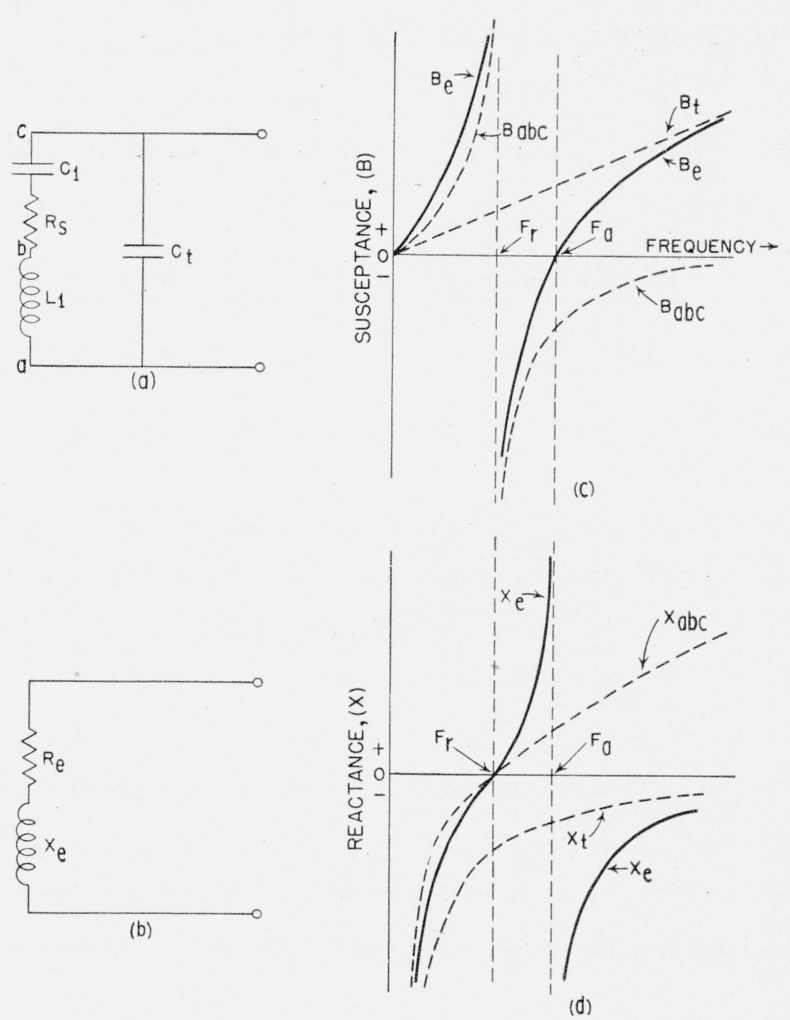

FIGURE 1.-Electrical equivalents of quartz-crystal units.

$a$. Equivalent circuit of quartz-crystal unit; $b$, effective impedance of a quartz-crystal unit between $f_{r}$ and $f_{a} ; c$, susceptance diagram of a crystal unit; $d$, reactance diagram of a crystal unit.

$R_{p}$ in some literature is referred to as the performance index $(P I)$. The equivalent termination impedance of the equivalent circuit of figure 1 may also be expressed in terms of the tuning shunt-capacity and the series resistance $R_{s}$ as follows: At any antiresonant frequency, $f_{a}$, (where $f_{a}>f_{\tau}$ ) the termination impedance is for practical purposes equal to that of a tapped antiresonant circuit and is resistive, namely,

$$
Z_{p}=R_{p}=Z_{x} \frac{C_{1}^{2}}{\left(C_{1}+C_{t}\right)^{2}}
$$

where

$$
Z_{x}=Q_{x} \frac{1}{\omega C_{e}}=\frac{1}{R_{s}\left(\omega C_{e}\right)^{2}} .
$$

$Q_{x}$ is the $Q$ of the antiresonant circuit, and $C_{e}=\left(C_{1} C_{t}\right) /\left(C_{1}+C_{t}\right)$.

$$
R_{p} \doteq \frac{1}{\omega^{2} C_{t}^{2} R_{s}}=\frac{X_{t}^{2}}{R_{s}}
$$


From eq 1 and 2

$$
\frac{X_{l}^{2}}{R_{s}} \doteq \frac{X_{e}^{2}}{R_{e}}
$$

$R_{p}$ may be plotted versus $C_{t}$ as an independent parameter in the form of a straight line having a negative 2 to 1 slope as shown in figure 4 . This follows from eq 2 as

$$
\log R_{p}=\log \left(\frac{1}{\omega^{2} R_{s}} \frac{1}{C_{t}^{2}}\right)=\log \frac{1}{\omega^{2} R_{s}}-2 \log C_{t} .
$$

$R_{s}$ is assumed to be a constant, and the percentage change in $\omega$ with $C_{t}$ is assumed to be negligible. These assumptions are justified on the basis of experimental evidence, as is shown elsewhere. Hence

$$
\log R_{p}=K-2 \log C_{t} .
$$

Similarly, the antiresonant frequency increments $\left(f_{a}-f_{r}\right)$ may be placed as a straight-line function of $C_{t}$ having a negative 45 degree slope, as shown in figure 4 . This may be shown as follows:

The resonance frequency is given by the relation (see fig. 1, a)

$$
\omega_{r}^{2}=\frac{1}{L_{1} C_{1}} .
$$

The antiresonance frequency is determined from the interrelation

$$
\omega_{a}^{2}=\frac{1}{L \frac{C_{1} C_{\iota}}{C_{1}+C_{\iota}}}=\frac{C_{1}+C_{t}}{L_{1} C_{1} C_{t}}
$$

from 5 and 6

$$
\frac{\omega_{a}^{2}}{\omega_{r}^{2}}=\frac{f_{a}^{2}}{f_{r}^{2}}=\frac{\left(C_{1}+C_{t}\right) C_{1}}{C_{1} C_{t}}=1+\frac{C_{1}}{C_{i}}
$$

or

$$
\frac{f_{a}^{2}}{f_{r}^{2}}-1=\frac{f_{a}^{2}-f_{r}^{2}}{f_{\tau}^{2}}=\frac{C_{1}}{C_{t}}
$$

Therefore,

$$
\frac{\left(f_{a}+f_{r}\right)\left(f_{a}-f_{r}\right)}{f_{r}{ }^{2}}=\frac{C_{1}}{C_{t}}
$$

For practical purposes, $\left(f_{a}+f_{r}\right)=2 f_{r}$. Hence

$$
\frac{2\left(f_{a}-f_{r}\right)}{f_{r}} \doteq C_{1} C_{t}^{-1},
$$

and

$$
\left(f_{a}-f_{r}\right) \doteq \frac{1}{2} f_{r} C_{1} C_{t}^{-1}
$$

or

$$
\log \left(f_{a}-f_{\tau}\right)=\log \left(\frac{1}{2} f_{r} C_{1}\right)-\log C_{t} .
$$

As $f_{r}$ and $C_{1}$ remain constant,

$$
\log \left(f_{a}-f_{r}\right)=K-\log C_{t},
$$

and the function of $\log \left(f_{a}-f_{\tau}\right)$ versus $\log C_{t}$ has a negative slope of 45 degrees.

Measurement results shown later (section IV) demonstrate how closely the straight lines and slopes were reproduced. The advantage of this method of presentation will also be apparent when the resulting curves are studied. A departure from either a straight line or from the proper slope usually indicated abnormally high series resistance or the proximity of an interfering closely coupled mode of vibration. Other information conveyed in this manner will be discussed later.

\section{Measurement Methods}

\section{Parameters Involved}

The following parameters were investigated in these measurements:

$C_{0}=$ internal capacity of a crystal unit with the crystal inactive.

$C_{t}=C_{0} \pm \Delta C_{t}=$ total effective capacity shunting the equivalent series resonant circuit of a crystal unit as shown in figure 1, a.

$\Delta C_{t}=$ an increment of $C_{t}$

$C_{1}=$ equivalent series (motional) capacity as shown in figure 1, a

$f_{r}=$ series resonance frequency of the crystal unit

$f_{a}=$ parallel resonance (antiresonance) frequency of the crystal-unit at any $C_{t}$.

$\Delta f=f_{a}-f_{r}$

$L_{1}=$ equivalent series inductance as shown in figure 1 , a

$R_{s}=$ series resistance of the crystal as shown in figure 1 , a

$R_{p}=$ equivalent antiresonance impedance (nonreactive) of the crystal unit corresponding to different values of $C_{t}$.

$R_{e}=$ equivalent series resistance of a crystal unit at any frequency, as shown in figure $1, b$ 
$R_{h}=$ resistance shunting the quartz crystal (equivalent to holder losses)

$Q_{e}=\frac{X_{e}}{R_{e}}=$ the effective " $Q$ " of the crystal unit

$Q_{x}=\frac{2 \pi f L_{1}}{R_{s}}=\frac{1}{2 \pi f C_{1} R_{s}}$ by definition, where $f$ is the nominal frequency of the crystal unit. This is usually referred to as the " $Q$ " of the crystal.

$X_{e}=$ the equivalent series (positive or negative) reactance of the crystal. $X_{t}=\left(1 / 2 \pi f C_{t}\right)$.

Some useful expressions of parameters in terms of the directly measurable quantities are derived as follows:

From eq 11 above,

$$
C_{1} \doteq \frac{2 \Delta f C_{t}}{f_{r}}
$$

Substituting this value into the defined expression of $Q_{x}$ and noting that $\Delta f \ll f_{r}$; i. e., that any frequency between $f_{a}$ and $f_{r}$ can be used for this expression.

$$
Q_{x}=\frac{f_{r}}{\left(2 \pi f_{\tau}\right) 2 \Delta f C_{t} R_{s}}=\frac{1}{4 \pi \Delta f C_{t} R_{s}} .
$$

Measurements of $f_{a}$ to determine $\Delta f$ are usually made at $C_{t}=C_{0}$, then

$$
Q_{x}=\frac{1}{4 \pi \Delta f C_{0} R_{s}} .
$$

At resonance

$$
L_{1}=\frac{1}{\left(2 \pi f_{\tau}\right)^{2} C_{1}} \cdot
$$

Equations 1 and 2 express $R_{p}$ in terms of $C_{t}$ and $X_{e}$.

The effect of holder losses reduces $R_{p}$ values to

$$
R_{p}^{\prime}=\frac{R_{h} R_{p}}{R_{h}+R_{p}} \cdot
$$

\section{Q-Meter Measurements}

It is noted above that the equivalent circuit of a crystal unit is a reactance $X_{e}$ in series with a resistance $R_{e}$ at all frequencies between $f_{r}$ and $f_{a}$. Numerous papers have shown the significance of these parameters in oscillators and filters. These parameters may be measured with laboratory instruments of which the $Q$-meter, twin- $T$, and impedance bridge were used here. These will be briefly analyzed.
The well-known Q-meter principle makes use of an antiresonant circuit arrangement, as shown in the table of figure 3 .

$L_{Q}, C_{Q}$ is tuned for antiresonance at the frequency at which $X_{e}, R_{e}$ is to be measured. The crystal unit is then connected across $C_{Q}$. It is evident that $C_{Q}$ has to be readjusted by $\Delta C_{Q}$ to restore the antiresonance at the $Q$-meter tank and that in general

$$
X_{\epsilon}=\frac{1}{\omega\left(\Delta C_{Q}\right)}
$$

At $f_{a}$ of the crystal unit corresponding to $C_{t}=C_{0}$, $\Delta C_{Q}$ will be zero; for lower and higher values of $f_{a}, \Delta C_{Q}$ will be correspondingly positive and negative.

The voltage across the antiresonance tank is a measure of the $Q$ of the circuit. Connecting the crystal unit across the tank reduces the original $Q$ as a result of the shunt effect of $R_{e}$. From the two values of $Q, R_{e}$ can be computed from

$$
R_{e}=\frac{1.59 \times 10^{8}\left(\frac{C_{Q 1}}{C_{Q 2}} Q_{1}-Q_{2}\right)}{f C_{Q 1} Q_{1} Q_{2}},
$$

where

$C_{Q 1}=$ the capacity, in micromicrofarads, required to tune the Q-meter tank without the crystal unit at the crystal frequency

$C_{Q 2}=$ the capacity, in micromicrofarads, required to tune the Q-meter tank with crystal unit in parallel with it

$Q_{1}=$ the original $Q$ of the tank

$Q_{2}=$ the reduced $Q$ of the tank

$f=$ the nominal frequency in $\mathrm{kc} / \mathrm{s}$ of the crystal unit.

The equivalent antiresonant impedance (nonreactive), $R_{p}$, of the crystal unit (PI) $[6,7]$ is the impedance of the crystal unit at a given frequency between $f_{r}$ and $f_{a}$ with the proper capacity connected in parallel with it.

The equations below state the values of $R_{p}$ and $R_{s}$ directly in terms of the $q$ 's observed.

$$
\begin{aligned}
& R_{p}=\frac{1.59 \times 10^{8} Q_{1} Q_{2}}{f C_{Q_{1}}\left(Q_{1}-Q_{2}\right)} \\
& R_{s}=R_{p}\left[\frac{\left(Q_{1}-Q_{2}\right) C_{Q_{1}}}{Q_{1} Q_{2}\left(C_{Q_{2}}-C_{Q_{1}}\right)}\right]^{2},
\end{aligned}
$$

where the terms are as stated for eq20. 
For high $R_{p}$ values, corrections are made for holder losses; the corrected $R_{p}^{\prime}$ value (from eq18) should then be used in eq22.

Measurements of $R_{p}$ values down to a few thousand ohms may be made with the $Q$-meter by using the above formulas. It was found, however, that a direct substitution method of using $\mathrm{r}-\mathrm{f}$ resistors eliminated time-consuming computations, without sacrificing accuracy.

A number of cpils (from 0.5 to $10 \mu h$ for the 8.7 Mc units) were tuned to the desired frequency, and their effective Q's were calibrated against r-f
Figure 2 shows a block diagram of the equipment. For low voltages (across the crystal units) a commercial Q-meter was used. Stable-frequency $r-f$ voltage was applied directly to the $0.04 \mathrm{ohm}$ resistance element in the Q-meter tank with the internal oscillator disconnected. This was done by leaving the ?-meter band switch in an intermediate position.

Some of the precautions observed while using the $Q$-meter are mentioned. Calibration curves were made with the external source, and the resistors and crystal units were connected across

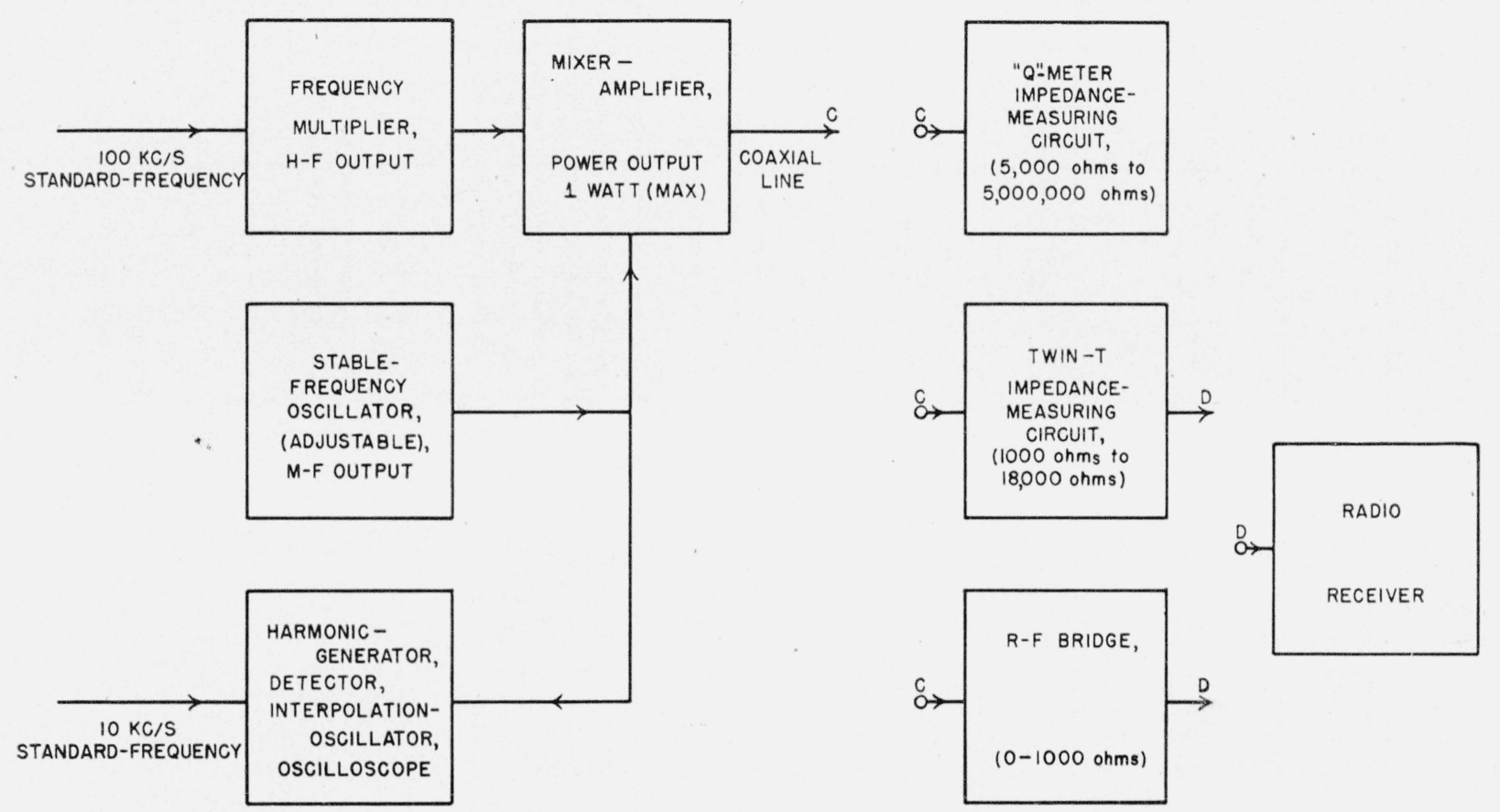

FIGURE 2.-Block diagram of the apparatus used in measuring the electrical characteristics of the quartz-crystal units.

resistors. The resistance values of the latter were checked from time to time with a d-c resistance bridge. These resistors were of the metallized-filament type enclosed in an isolantite body. The discrepancy between the $d-c$ and $r-f$ values of these resistances and the degree with which the $Q$-meter values can be read and reproduced largely determine the accuracy of these $Q$-meter measurements. Conservative estimates of the over-all accuracy are tabulated in figure 3 . The inductance and the $Q$ of an individual coil determine its resistance range for greatest accuracy; this is readily apparent when calibration curves of $R_{p}$ 's are plotted against $Q_{2}$ on semilog paper with resistance on the logarithmic coordinate. the $Q$-meter tank with shortest leads practicable. Care was taken to eliminate all direct pick-up in the coils. Plug-in arrangements were such that no additional losses were introduced across the crystal units. Inaccuracies in measurements as a result of $Q$-meter limitations were often checked. Among these was the effect of the " $Q$ " level on values of $C_{0}$ usually measured with the $Q$-meter (using the internal oscillator at a frequency considerably below the nominal crystal frequency). The current level in the 0.04-ohm resistor was maintained constant for a given zero setting of the " $Q$ "-indicator. Values of external capacitors used as $\Delta C_{t}$ were measured at the nominal frequency of the crystal units. 


\begin{tabular}{|c|c|c|c|c|c|c|}
\hline PARAMETER & RANGE & INSTRUMENT & $\begin{array}{l}\text { CIRCUIT ARRANGEMENT } \\
\text { (FUNDAMENTAL SCHEMATIC) }\end{array}$ & PROCEDURE & PRECAUTIONS & $\begin{array}{l}\text { ACCURACY } \\
\text { CONERVATVE } \\
\text { ESTIMATE }\end{array}$ \\
\hline \multirow{4}{*}{\begin{tabular}{c|} 
STATIC \\
CAPACITY \\
$C_{0}$ \\
AND \\
PARALLELING \\
CAPACITY \\
$\triangle C_{t}$
\end{tabular}} & O то $30 \mu \mu \mathrm{f}$ & \multirow{3}{*}{ "Q" METER } & \multirow{3}{*}{ SEE $f_{a}$} & \multirow{3}{*}{$\begin{array}{l}\text { CALIBRATE } c_{0} \\
\text { Vs. DIAL SETTING. } \\
C_{0}=c_{Q_{1}}-C_{O_{2}}\end{array}$} & \multirow{3}{*}{$\begin{array}{l}\text { MEASURE CO AT r.f. FOR } \\
\text { WHICH CRYSTAL IS INACTIVE } \\
\text { POSITION ANO SHIELONG } \\
\text { OF CRYSTAL UNIT IS IMPORTANT }\end{array}$} & $\pm 0.1 \mu \mu f$ \\
\hline & 30 To $150 \mu \mu f$ & & & & & $\pm 0.2 \mu \mu f$ \\
\hline & 150 Tо $2000 \mu \mu f$ & & & & & $\pm 5 \mu \mu f$ \\
\hline & 100 to $1100 \mu \mu f$ & $\begin{array}{l}\text { TWIN-T } \\
\text { IMFEDANCE-MEASURING } \\
\text { ClRCUIT }\end{array}$ & SEE $R_{p}$ & CALIBRATE CAPACITANCE DIAL & & $\pm 2 \mu \mu f$ \\
\hline \multirow{2}{*}{$\begin{array}{c}\text { SERIES - } \\
\text { RESONANCE } \\
\text { FREQUENCY, } \\
\mathrm{fr}_{\mathrm{r}}\end{array}$} & "Q" METER & "Q" METER & 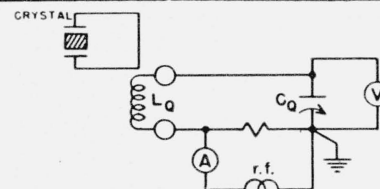 & $\begin{array}{l}\text { ADJUST } L_{0} C_{Q} \\
\text { FOR MAXIMUM V. } \\
\text { ADJUST r.f. } \\
\text { FOR MINIMUM V. }\end{array}$ & $\begin{array}{l}\text { USE SHORTED TERMINALS } \\
\text { OR SMALLEST LOQP } \\
\text { FOR INDUCTIVE COUPLING } \\
\text { TO CRYSTAL UNIT }\end{array}$ & $\pm 1.5 \mathrm{IN} 10^{6}$ \\
\hline & $R-F$ BRIDGE & R-F BRIDGE & SEE $R_{S}$ AND $R_{S}$ (HIGH LEVEL) & $\begin{array}{l}\text { ADJUST } r \text {. } f \text {., AND RESISTANCE ARM } \\
\text { OF BRIDGE FOR BALANCE }\end{array}$ & \begin{tabular}{|} 
SHIELD WELL. NO F.M. \\
IN OUTPUT OF $r$-f GENERATOR
\end{tabular} & $\pm 1.5 \mathrm{NN} 10^{6}$ \\
\hline \multirow[t]{2}{*}{$\begin{array}{c}\text { ANTIRESONANCE } \\
\text { FREQUENCY, } \\
f_{0}\end{array}$} & "Q" METER & "O" METER & 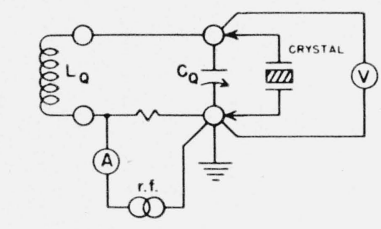 & $\begin{array}{l}\text { TUNE "Q" METER TO } f_{r} \text {. PLACE CRYSTAL } \\
\text { UNIT IN PARALLEL WITH C C. INCREASE } \\
\text { rf. FOR MAX.V. REMOVE CRYSTAL UNIT } \\
\text { AND RE TUNE "Q" METER. REPLACE } \\
\text { CRYSTAL UNIT AND READJUST r.f. } \\
\text { FOR MAX. V. }\end{array}$ & $\begin{array}{l}\text { FOR MAXIMUM PRECISION } \\
\text { ADJUST C C FOR MAX.V. } \\
\text { USE LARGE L/C RATIO } \\
\text { IN"Q" METER }\end{array}$ & $\pm 2.5 \mathrm{IN} 10^{6}$ \\
\hline & TWIN-T CIRCUIT & TWIN-T CIRCUIT & SEE $R_{p}$ (LOW LEVEL) & $\begin{array}{l}\text { ADJUST } r . f \text {. AND } C_{Q} \text { ARM } \\
\text { OF BRIDGE FOR BALANCE }\end{array}$ & SHIELD WELL & $\pm 2.5 \mathrm{iN} 10^{6}$ \\
\hline \multirow{2}{*}{$\begin{array}{c}\text { EQUIVALENT } \\
\text { 3ERIES RESISTANCE } \\
R_{0} ; \text { AND } \\
\text { REACTANCE, } \mathrm{X}_{0}\end{array}$} & O TO 1000 OHMS & \multirow{2}{*}{ R-F BRIDGE } & \multirow{2}{*}{ SEE $R_{S}$} & \multirow{2}{*}{ ROUTINE } & \multirow{2}{*}{$\begin{array}{l}\text { SHIELD WELL. USE LOW } \\
r-f \text { INPUT TO BRIDGE }\end{array}$} & $\begin{array}{l} \pm 0.2 \text { OHMS } \\
\pm 2 \%\end{array}$ \\
\hline & О TO 500 OHMS(AT IO MC.) & & & & & $\begin{array}{l} \pm 1 \text { OHM } \\
\pm 1 \%\end{array}$ \\
\hline
\end{tabular}

Figure 3.-Outline of measurement methods used. 


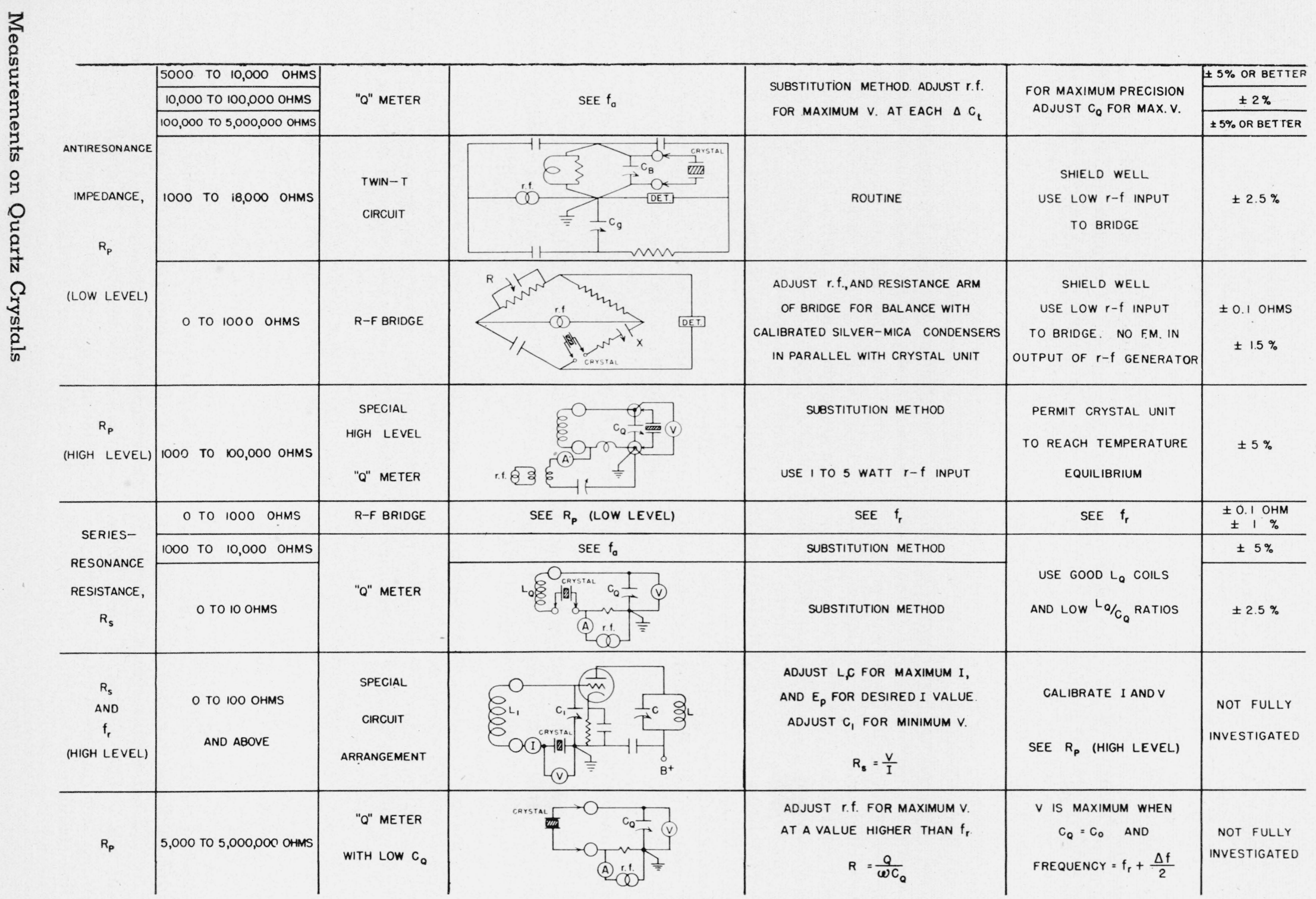

FIGURE 3.-Outline of measurement methods used. 
The resonance and antiresonance frequencies $\left(f_{r}\right.$ and $\left.f_{a}\right)$ were most readily determined with the $Q$-meter. An unshielded coil was resonated in the $Q$-meter at a frequency near that of the crystal unit. The crystal unit was short-circuited and placed near the low voltage end of the coil. When the Q-meter frequency was then varied, a sharp dip appeared on the $Q$-indicator at that frequency equal to the $f_{r}$ of the crystal unit. As the value of $f_{r}$ is affected by any inductance or capacitance connected in the short-circuiting path, precautions were taken to minimize this in precise measurements.

Measurements of $f_{a}$, (when $C_{t}=C_{0}$ ), were made with the crystal unit connected in parallel with the Q-meter coil. The coil was first resonated at the nominal crystal-unit frequency. After the insertion of the crystal unit, the frequency was varied until the $Q$-indicator rose to a maximum. With the crystal unit again removed, the coil was resonated at constant frequency by adjusting the variable capacitor $\left(C_{Q}\right)$ of the $Q$-meter. Once again the crystal unit was placed in the circuit and the frequency adjusted to a maximum $Q$ indication. These adjustments were repeated until a constant, frequency value $\left(f_{a}\right)$ was obtained. At this point $C_{t}=C_{0}$.

To measure $f_{a}$ when $C_{t}$ is not equal to $C_{0}$, the Q-meter capacitance was changed from the $C_{t}=C_{0}$ setting by the required $\Delta C$, and the frequency was varied to a new $f_{a}$, as indicated by the peaking of the $Q$-indicator. It is evident that the sharpness of the $f_{r}$ and $f_{a}$ indications depends upon the $L / C$ ratio of the $Q$-meter tank.

Measurements of $R_{p}$ values corresponding to given frequencies within a response were made by using the substitution method. For highest precision, a final adjustment of $C_{Q}$ to a maximum $Q$ indication was made at constant frequency. Measured $Q$ values were converted into corresponding $R_{p}$ values from the $R_{p}$ versus $Q_{2}$ calibration curves. $R_{p}$ was computed from $C_{t}$ and $R_{s}$, for $R_{p}$ values low enough to be unaffected by holder losses.

Values of $R_{p}$ at voltages closely approaching those applied to the crystal unit in a Miller- (or Pierce-) type oscillator were determined with the specially constructed circuit shown in figure 3 . This circuit was similar to that of the $Q$-meter, except that the voltage was injected into the $L-C$ tank by link-coupling the output of an auxiliary amplifier into a very small section of the tank inductance. The substitution method was again used. The current- and $Q$-indicators of the regular Q-meter were replaced by sensitive thermoelements with potentiometer-indicators. Great care had to be observed in these measurements to avoid the frequency response shift, as the temperature of the crystal unit varied with the high current through it. The procedure was modified to enable presetting the voltage values. desired. Since, as is shown below, there was no apparent difference in the $R_{p}$ values at high and low voltages, there seems to be no need of measuring crystal parameters at high voltages. The procedure is therefore not elaborated upon.

The $Q$-meter was used for measurements of $R_{s}$ values directly. The crystal units were connected in series with the tank coil for low values of $R_{s}$ (up to $10 \mathrm{ohms}$ ) and in parallel with the tank for values higher than $1,000 \mathrm{ohms}$. It was found, however, that an $r$ - $f$ impedance bridge was more suitable for measurement of $R_{s}$ values from 1 to 1,000 ohms. This is described later in greater detail.

It should be mentioned here that when a crystal unit is connected across a tank tuned approximately to the crystal frequency there are present two antiresonance peaks of the circuit impedances [5]. As the lower peak is not governed by the frequency response of the crystal unit, and as it appears at a separation many times the width of $\Delta f$, it does not affect the method of measurement.

\section{Twin-T Circuit and Impedance Bridge}

A twin- $T$ admittance measuring circuit was used for $R_{p}$ values of 1,000 to 10,000 ohms. As this is an admittance measuring device, individual resistance values were computed from the results obtained and were occasionally checked against measurements of carbon-film resistors. Care was taken that increments of $C_{t}$ applied across the crystal units (either by varying the internal capacity of the instrument or by connecting external condensers across them) were known to the greatest accuracy at the operating frequency. The fundamental circuit is similar to that of the $Q$-meter and the over-all accuracies were the same as with the $Q$-meter.

It is possible to measure $X_{e}$ and $R_{e}$ values of a crystal unit directly with the twin- $T$. However, the relatively limited range of the measurable $X_{e}$ 
at corresponding $R_{e}$ values renders the instrument useful only in double-checking a few points obtainable with the Q-meter, and its use yields little additional information.

A commercial $r-f$ bridge was used to measure $R_{s}, R_{e}$ and $X_{e}$ values from 1,000 down to a few ohms, and $R_{p}$ values down to 100 ohms. In fact, $R_{p}$ values as low as 10 ohms were actually measured with the 8.7-Mc crystal units. However, the validity of the latter was questioned.

The major requirement in the application of the twin- $T$ and impedance bridge was a stable frequency source, adjustable in small increments of frequency. With such a source and the usual precautions for proper shielding and grounding of the measuring and detecting equipment, no difficulties were encountered in following the normal bridge operation procedures. A source of radio frequency having good waveform without frequency modulation was found essential for a sharp null balance.

The general procedure in measuring $R_{s}$ and $R_{p}$ with the $r-f$ bridge was to vary the frequency and the resistance arms until a minimum deflection on the null balance indicator was obtained. The value of $R_{s}$ was measured more rapidly by first determining $f_{r}$ with the $Q$-meter, as explained above.

$R_{p}$ values were measured with external capacitors connected in shunt with the crystal-unit terminals. $R_{e}$ and $X_{e}$ values were obtained at any frequency by adjusting the resistance and reactance arms for a null balance.

It was found advisable to use the lowest input voltage to the bridge required for effective operation of the detector. At low-input levels no appreciable heat was generated inside the crystal. Consequently, frequency drift was minimized. Good correlation was then obtained between parameters and frequency values within a given $\Delta f$.

The accuracy of the $r-f$ bridge in all measurements is shown in figure 3 .

\section{Secondary Responses}

The adjustable frequency range available at the time to explore the spectrum for secondary (spurious) responses limited these measurements to the 8.7-Mc crystal units within a band of 8650 to $8892.5 \mathrm{kc} / \mathrm{s}$. This range is in the process of being extended for wider investigation in the future.
In locating and measuring secondary responses the Q-meter was singularly useful. The Q-meter tank was tuned to a frequency somewhat lower than the nominal frequency of the crystal unit. The frequency was then continuously increased, with the crystal connected across the tank. Voltage dips were then observed at $f_{r}$ points and peaks at $f_{a}$ points in the frequency spectrum. Individual responses were closely explored by retuning the tank (with crystal unit removed) and repeating the sweep of frequency to determine more exactly the values of $f_{r}$ and $f_{a}$. The process of retuning the tank and of readjusting the frequency was repeated until no differences in frequency values of $f_{\tau}$ or of $f_{a}$ were noticed.

High-inductance tank coils were more effective in locating $f_{a}$ values; similarly, low-inductance coils were more effective in locating $f_{r}$ values. It was sufficient in most cases to use a coil of medium inductance to serve both purposes. For most accurate measurements of the frequencies and equivalent $R_{p}$ or $R_{s}$ values, different coils were used as conditions warranted.

It was found convenient and enlightening to plot the relative position of the secondary responses, as shown in figures 8 and 14. For practical purposes it might be sufficient to interconnect the $R_{s}$ and $R_{p}$ values of each response with a straight line as the curvature involved is negligible relative to the frequency scale employed. This approximation may be justified analytically when considering the variation of $R_{e}$ within a single response, between $f_{r}$ and $f_{a}$. Designating the total series reactance of the equivalent circuit of figure 1 , a, as

$$
X_{1}=\omega L_{1}-\frac{1}{\omega C_{1}} \text { and } X_{0}=\frac{1}{\omega C_{0}} \text {, }
$$

the termination impedance of the unit is

$$
Z=\dot{X}_{0}\left\{\frac{X_{0} R_{s}+j\left[R_{s}{ }^{2}+X_{1}\left(X_{1}+X_{0}\right)\right]}{R_{s}^{2}+\left(X_{1}+X_{0}\right)^{2}}\right\}
$$

then

$$
|Z|=X_{0} \frac{\left(X_{0}{ }^{2} R_{s}{ }^{2}+\left[R_{s}{ }^{2}+X_{1}\left(X_{1}+X_{0}\right)\right]^{2}\right)^{\frac{1}{2}}}{R_{s}^{2}+\left(X_{1}+X_{0}\right)^{2}} .
$$

The equivalent series values of $R_{e}$ and $X_{e}$ are then

$$
\begin{gathered}
R_{e}=\frac{X_{0}^{2} R_{s}}{R_{s}^{2}+\left(X_{1}+X_{0}\right)^{2}} \\
X_{e}=\frac{X_{0}\left[R_{s}^{2}+X_{1}\left(X_{1}+X_{0}\right)\right]}{R_{s}^{2}+\left(X_{1}+X_{0}\right)^{2}} .
\end{gathered}
$$


For practical purposes, at $f_{a}$ the reactive component of $Z$ vanishes and $X_{1}+X_{0}=0$. Therefore,

$$
Z_{a} \doteq R_{p} \doteq \frac{X_{0}{ }^{2} R_{s}}{R_{s}{ }^{2}}=\frac{X_{0}{ }^{2}}{R_{s}} \doteq R_{e}^{\prime},
$$

where $R_{e}^{\prime}$ is the value of $R_{e}$ at $f_{a}$. At $f_{r}, X_{1}=0$ and

$$
|Z|=X_{0} \frac{\left[X_{0}^{2} R_{s}{ }^{2}+R_{s}^{4}\right]^{\frac{1}{2}}}{R_{s}^{2}+X_{0}^{2}}=\frac{X_{0} R_{s}}{\sqrt{X_{0}^{2}+R_{s}^{2}}} \cdot
$$

For values of $X_{0} \gg R_{s}$,

$$
|Z| \doteq R_{s} .
$$

Similarly, at $f_{r}, X_{1}=0$, and from eq. 25,

$$
R_{e}=\frac{X_{0}^{2} R_{s}}{R_{s}^{2}+X_{0}^{2}} \doteq R_{s} \text {. }
$$

It is therefore apparent that for responses having series-resistance values considerably lower than $X_{0}$, plotting $R_{e}$ versus frequency, as shown in figures 8,14 , and 22 , would closely represent a quantitative variation of a single parameter of a crystal unit within the limit of $R_{s}$ and $R_{p}$. For responses having high series-resistance values, this presentation of a response is correct only at the $R_{p}$ and $R_{s}$ points. The intermediate points have qualitative significance only. It should be pointed out, however, that the $R_{p}$ and $R_{s}$ values representing the top and bottom points of each response are the actual measured values of these parameters. The $Q$-meter was used to locate the response, and $R_{s}$ and $R_{p}$ values were then measured with $Q$-meter, twin- $T$, or $r$ - $f$ bridge, depending on the magnitude of the parameter. The initial balance of the bridge and of the twin- $T$ circuit had to be made at the frequency and at the chosen voltage input value of the measurement performed at the time. Not all the relatively small secondary responses (having $R_{s}$ values above approximately $1,000 \mathrm{ohms}$ ) behave alike. Some of these appear to have $R_{p}$ values considerably lower than their $R_{s}$ values. Further study of their behavior will be required. Their presence is therefore indicated by a single point representing measured $R_{s}$ values. One may also assume that the small responses barely detectable with the Q-meter may be neglected.

\section{Notes on Methods and Equipment Employed}

The equipment shown in figure 2 was used for 8.7-Mc crystal measurements. A standard fre- quency of $100 \mathrm{kc}$ was fed into a frequency multiplier, the products of which were mixed with the output of a continuously adjustable stable-frequency oscillator. The output of the mixer was then amplified to the desired level.

Frequency could be easily adjusted, measured, and maintained to better than 5 parts in 10 million over a period required to make any one measurement. Regular frequency measuring equipment incorporating a radio receiver as a detector, a stable radio-frequency oscillator to determine the beat frequency between the adjustable oscillator and a harmonic of the standard source, and an oscilloscope were used throughout these measurements. A second radio receiver was used as a null indicator with the twin-T and $r-f$ bridge.

Equipment employed in the measurements of the 50- and 100-ke crystals was essentially the same.

Special circuits were developed and satisfactorily used to measure independently and directly the values of $R_{s}$ and $f_{r}$. For convenient reference, figure 3 outlines briefly the methods, accuracy, and range of parameters measured.

\section{Graphical Presentation and Results of Measurements}

\section{Characteristics of Normal-Crystal Unit}

A crystal unit operating at a frequency response with proper slopes of $R_{p}$ and $f$ versus $C_{t}$ is designated as a normal crystal. Figures 4 to 11 show characteristics of normal crystal units. Slopes of $2: 1$ and $1: 1$ were plotted for the straight-line sections in figures 4 to 7 . The position of the points show the departure of measurement data from the theoretical slopes. Curvatures at high $R_{p}$ values were caused by holder losses, as evidenced by some of these characteristics measured with the crystal removed from the holders or when placed in low-loss holders. The very low $R_{p}$ region at these crystal units was not fully investigated.

The displacement of the $R_{p}-C_{t}$ curve shown in figure 5 indicates a drop in the $R_{p}$ values over the entire range of $C_{t}$. This apparently resulted from an increased $R_{s}$ value because of an accumulation of additional dust particles on the crystal plate when it was removed from the holder. It is seen, however, that a $2: 1$ slope is maintained throughout the curve up to $C_{t}-C_{0}$. 


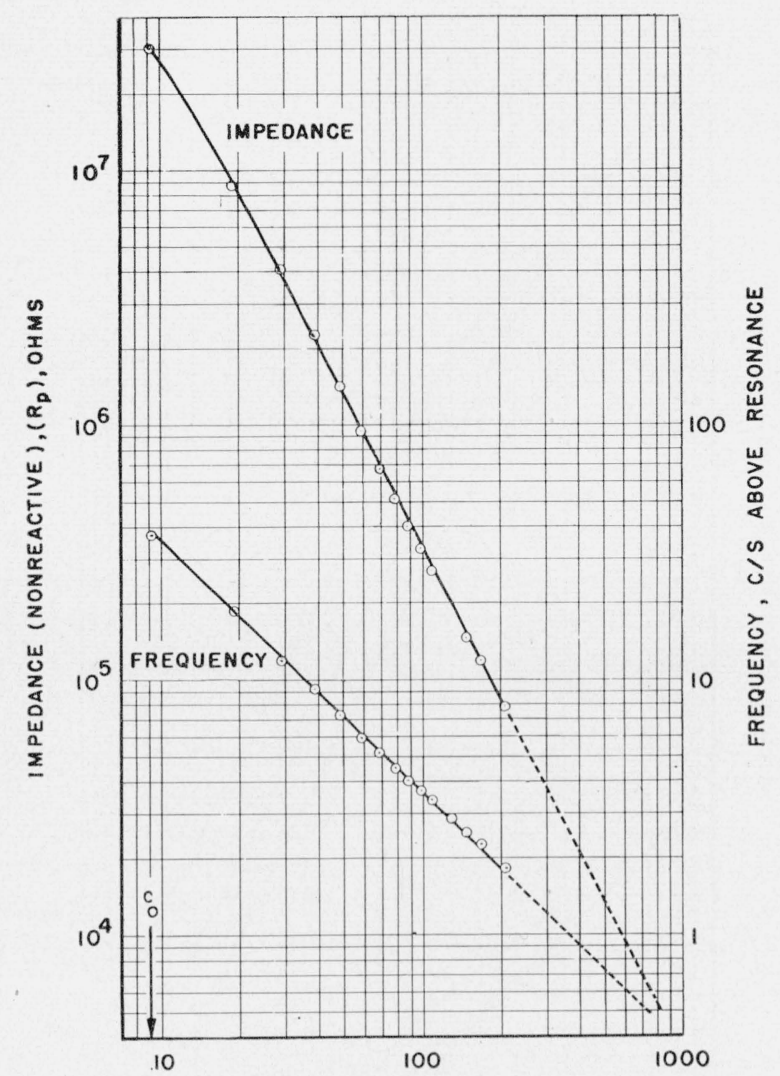

$c_{t}$, NNf (CAPACITY $c_{0}$, PLUS EXTERNAL PARALLELING CAPACITY)

FiguRE 4.-Electrical characteristics of a 50-kc quartz bar: $X$-cut, metal-film electrodes, clamp-type mounting.

Figure 6 shows these characteristics for two plated wire-supported B'T-cut 8.7-Mc units. Curves of four other units of the same type were obtained, and, if plotted, would fall between these two sets of curves. A wider variation of $R_{p}$ values is shown in figure 7 , which represents three unplated and one plated pressure-mounted, BTcut, 8.7-Mc units. The $R_{p}$ and frequency points of two of the unplated units fall within the curve $A$. The frequency curves of the unplated units deflect from a 45-degree slope as $C_{\imath}$ approaches $C_{0}$. It is felt, however, that this may not constitute a real departure from the normal condition. Further investigation should answer this question.

The frequency spectrum of one of the wiremounted $8.7-\mathrm{Mc}$ units is shown in figure 8 . It closely represents the spectrum of every one of this group of six units, both in relative position and in the intensity of the responses. This spectrum shows at a glance the relative freedom of the lowest frequency response from neighboring interfering responses, and should be compared with the spectrum of an unplated unit shown in figure 14 .

It seems that two sets of curves similar to those shown in figures 6 and 8 may effectively demonstrate the electrical characteristics and behavior of a crystal unit. This might be more apparent when the curves are compared with those obtained for complex units shown later.

It might seem, at first, as if a single point on the $R_{p}-f-C_{t}$ curve through with the engineer could draw the appropriate $2: 1$ or $1: 1$ line would convey sufficient data for design purposes. However, the complete $R_{p}-f-C_{t}$ curves are necessary to show the effect of holder losses, and the actual deviation of the slopes from normal (whatever the cause); while the frequency spectrum is required to show

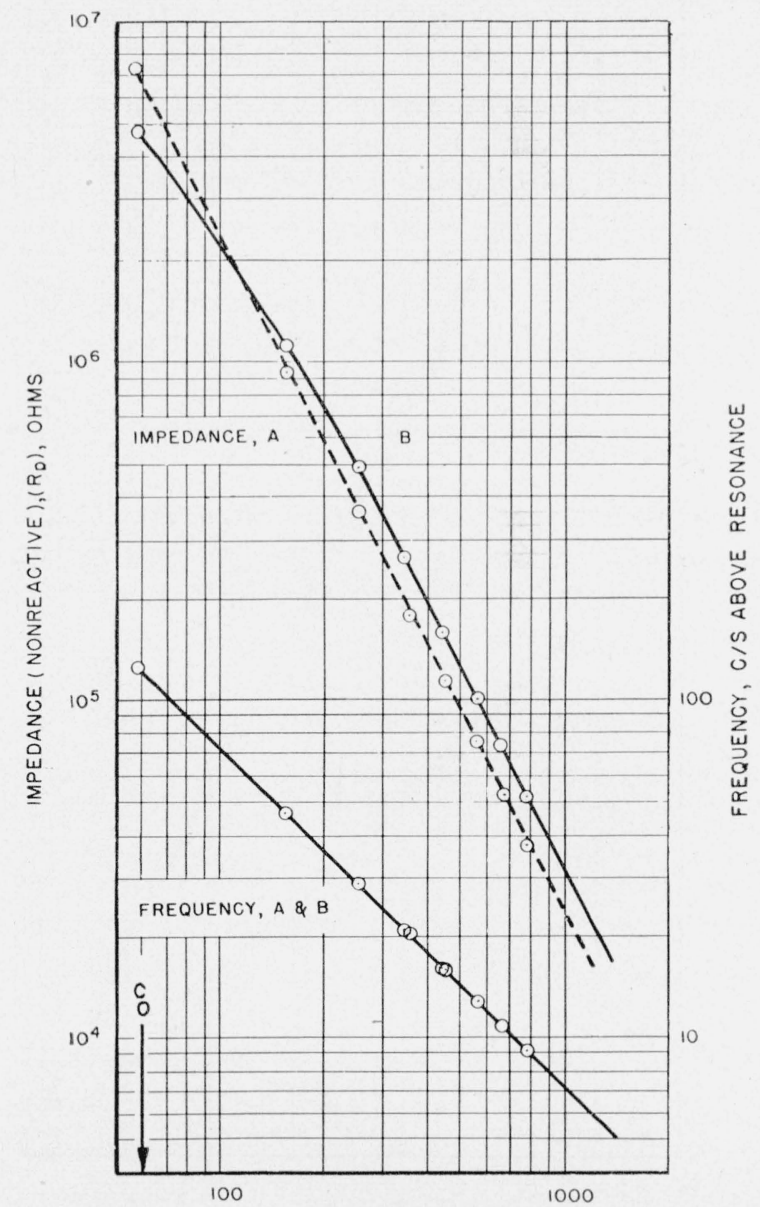

$c_{t}$, $\mu \mu f$ (CAPACITY $C_{0}$, PLUS EXTERNAL PARALLELING CAPACITY)

FIGURE 5.-Electrical characteristics of a 100-kc quarizcrystal unit: GT-cut, metal-film electrodes, wire-mounted crystal, and evacuated container. 
possible operation of the crystal unit at more than one frequency.

$R_{s}$ could be computed from eq 2 for any value of $R_{p}$ and the corresponding $C_{t}$ on the straight portion of the curve; however, the value of $C_{o}$ would have to be measured or supplied by the manufacturer. In short, a set of curves, as shown in figures 6 and 8 , supplies all the essential equivalent electrical characteristics of a crystal unit when either $f_{r}$ or $f_{a}$ at $C_{o}$ is also given. $L, C$, and $Q_{x}$ of the crystal unit can be determined from eq $11,14,15$, and 16 as $C_{o}, \Delta f$, and $R_{s}$ are known. On the other hand, a set of crystal-unit data omitting any of these fundamental parameters (i. e., $C_{o}, \Delta f, R_{s}$, and $f_{r}$ or $f_{a}$ ) seems to be inadequate in stating the relative merit of a quartzcrystal unit. It is here assumed that data on

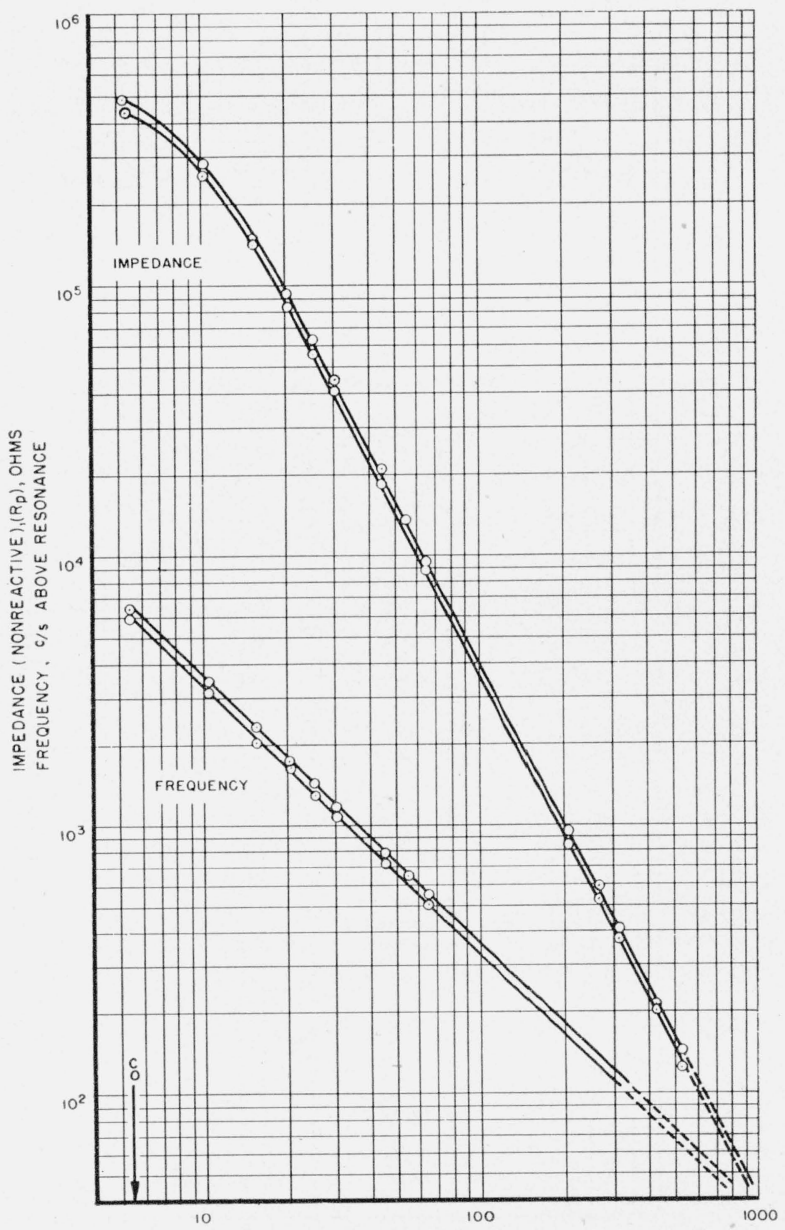

$C_{t}, \mu \mu f$ (CAPACITY $C_{0}$, PLUS EXTERNAL PARALLELING CAPACITY)

FIGURE 6.-Electrical characteristics of two type $C R-1$ crystal units: BT-cut, metal-film electrodes, wire-mounted crystal, plastic container.



FiguRe 7.-Electrical characteristics of four "normal" 8.7$M c$ crystal units $(B T-c u t)$.

$A$, Represents two crystals having almost identical impedance and frequency characteristics. Both are air-gap, pressure mounted: one a CR-1, the other an FT-243 type; $B$, FT-243 crystal unit with metal-film electrodes and spring-clip mounting; $C, \mathrm{FT}-243$ erystal unit with air-gap electrodes and pressure mounting.

parameter variations with temperature are always indispensable.

Figure 9 shows the main (oscillator-controlling) response (curve $A$ ) of an unplated $8.7-\mathrm{Mc}$ unit and the response (curve $C$ ) adjacent to it, the two having an $f_{a}$ separation of 2,990 cycles. Placing the same crystal in a polystyrene holder extended the straight portion of the $R_{p}$ curves ( $B$ and $D$ ) to values of $C_{t}$ beyond $C_{0}$. These data were taken primarily in an attempt to separate holder losses from losses that might conceivably be coupled 
into the first response by the proximity of a second response. It may be seen that up to $C_{t}=C_{o}$ the departure of the $R_{p}$ curve below $C_{o}$ the sharpness of the peak of the $R_{p}$ curve as well as the high $R_{p}$ values of the adjacent response suggest little coupling effect.

Equivalent series resistance and reactance values at frequencies above and below resonance are shown in figure 10 for one of the normal, plated, wire-mounted $8.7-\mathrm{Mc}$ units. The measurements were made with the $r-f$ bridge, which limited the range to the values shown. These curves could be extended to higher positive values of $X_{e}$ by the use of the Q-meter data and eq1. No attempt was made at this time to measure higher negative values of this parameter.

It was of interest to compare the behavior of the parameters of a secondary and primary response of a normal crystal (with the two responses widely separated). The $R_{p}$ and frequency versus $C_{t}$ curves of a large secondary response of one of the wire-mounted units are shown in figure 11 . A considerable departure from a 1:1 slope may be observed in the frequency curve, whereas the $R_{p}$ curve seems to have a normal slope.

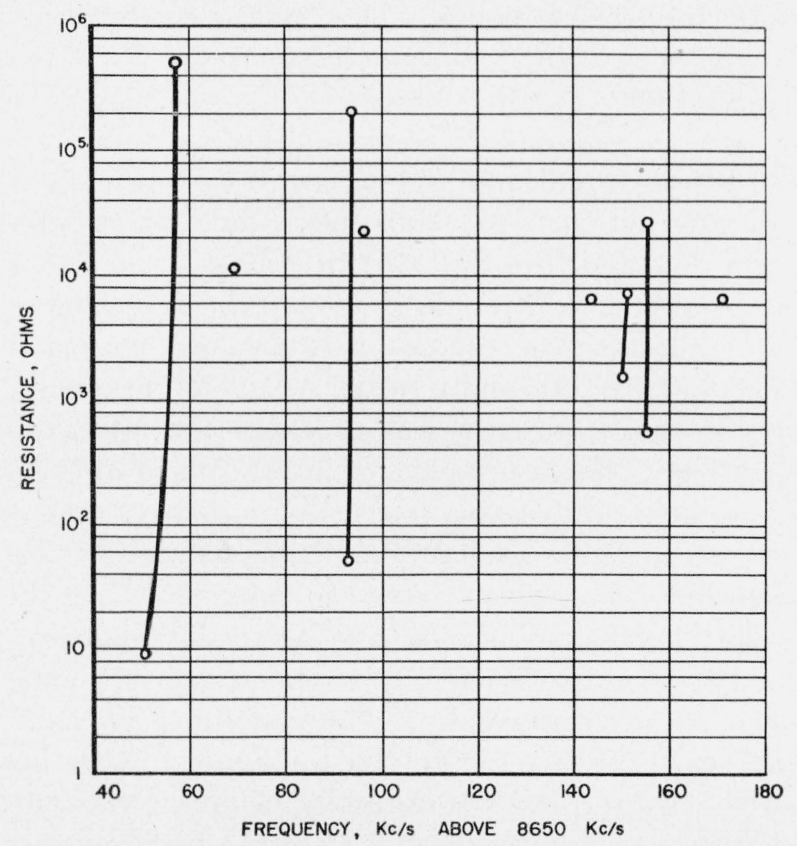

FugURE 8.-Frequency-response spectrum of a metal-film electrode, wire-mounted crystal unit of the CR-1 type.

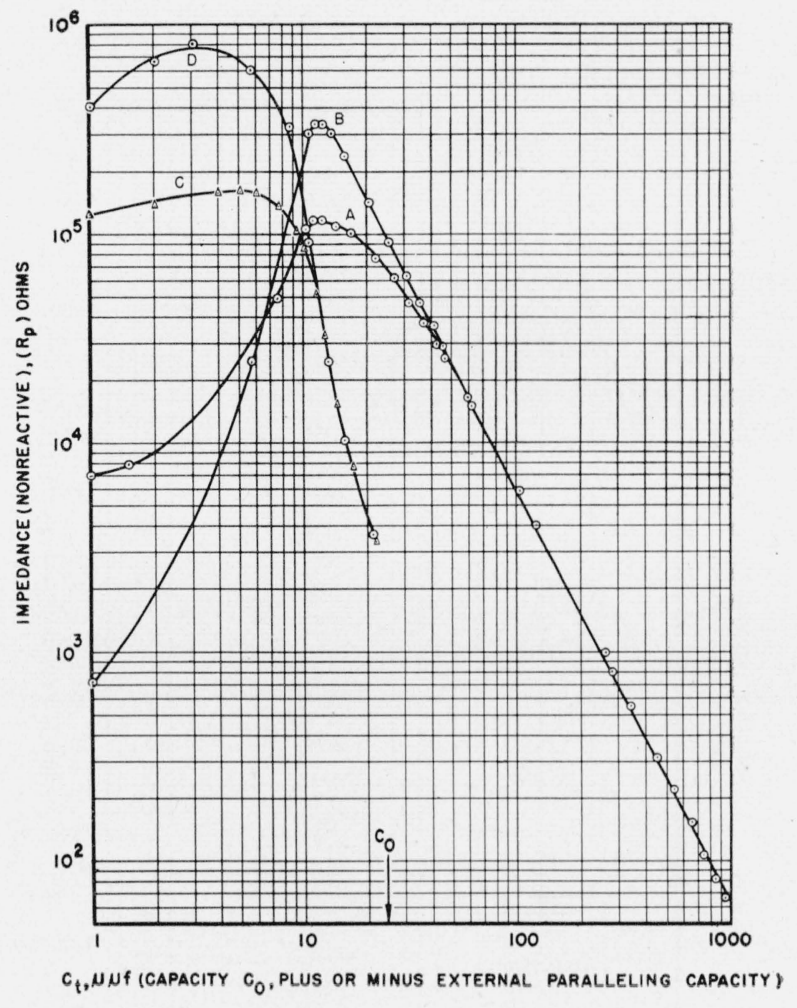

Figure 9.-Impedance measurements above and beiow antiresonance of the first two adjacent responses of an air-gap, pressure-mounted crystal unit of the FT-243 type: 8.7 $M c, B T$-cut.

$A$, First response (lowest frequency), plastic container; $B$, first response: special low-loss polystyrene container; $C$, second response: plastic container; $D$, second response: special low-loss polystyrene container.

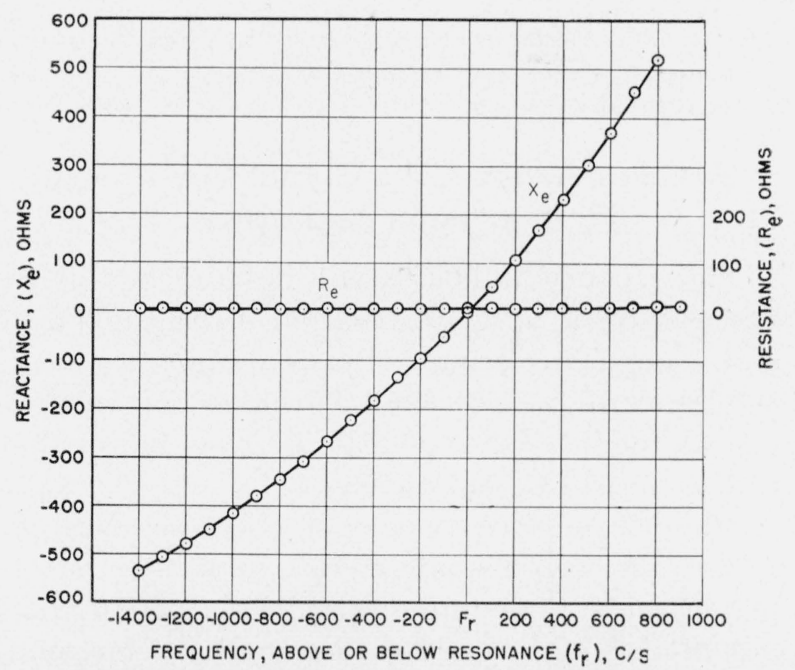

Figure 10.-Electrical characteristics above and below series resonance of a crystal unit of the CR-1 type: BT-cut, 8.7-Mc, metal-film electrodes, wire mounted. 


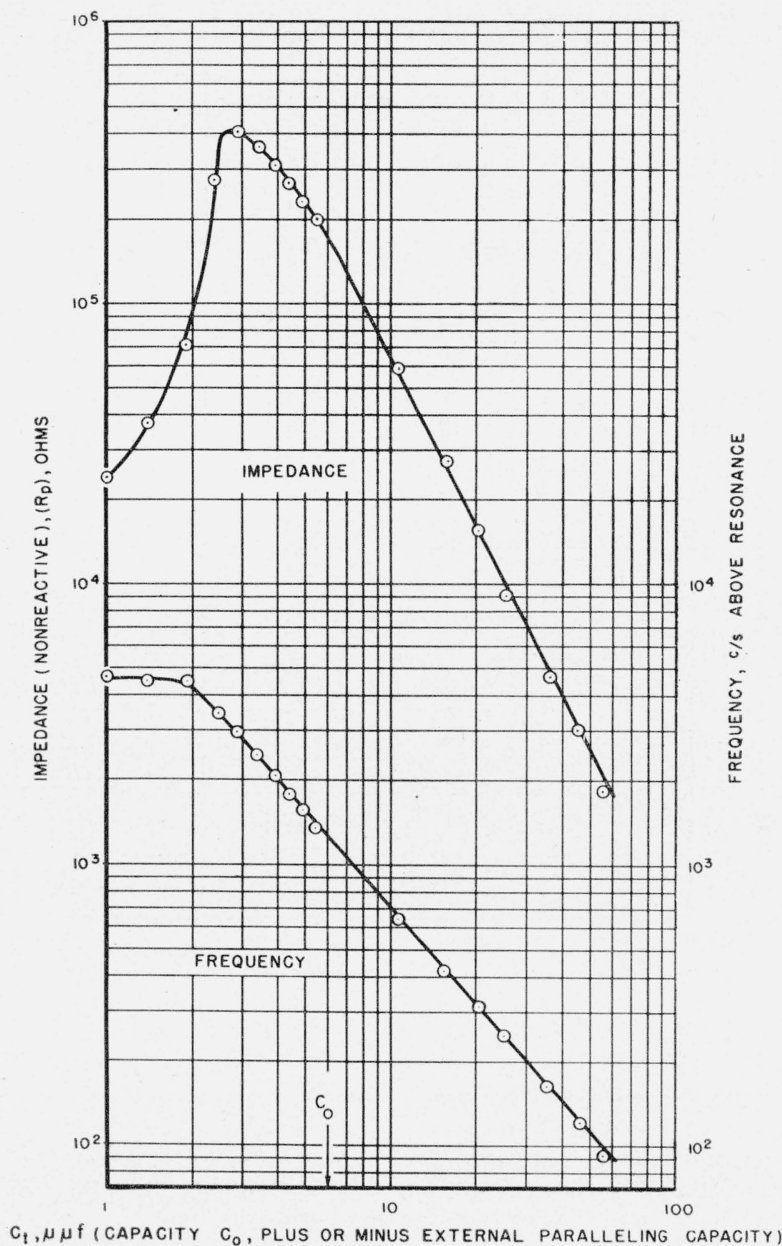

Figure 11.-Electrical characteristics above and below antiresonance of the second large response of crystal unit described in figure 10.

\section{Characteristics of a Crystal Unit Having Interfering Responses}

The greatest difficulty encountered in this investigation, as already mentioned, was to locate and to segregate groups of crystal units, the characteristics of which could be measured and the results reproduced at will to an accuracy of that of the measuring equipment.

Stability of activity and of $R_{s}$ values was observed on approximately 100 unplated, on 25 plated pressure-mounted, and on six plated wiremounted 8.7-Mc crystal units. Daily measurements were made over a period of weeks. The most stable units were segregated successively by further observations in other equipment. For example, some units were rejected when instability of $R_{p}$ values was observed in the $Q$-meter.
It was not intended at the time to study the causes of the instability, which, as may be realized, is a separate problem. Therefore, figure 12 is shown merely to demonstrate the relative instability encountered and the minimum stability required for reliable measurements. It may be noted that all the wire-mounted units were equally stable, whereas approximately 10 percent of the others were stable to the same degree. Some of the units relatively stable with respect to $\mathrm{I}_{g}$ and $R_{s}$ had $R_{p}$ and $f$ versus $C_{t}$ characteristics deviating considerably in slope from the expected $2: 1$ and $1: 1$ ratios. This is shown in figure 13 . It is seen here that for $C_{t}$ values between 75 $\mu \mu f$ and $C_{o}$, the first response has higher $R_{p}$ values than the second response. Above $75 \mu \mu f$ the $R_{p}$ values of the second response are higher. Thus at a $C_{t}$ of approximately $75 \mu \mu f$, this unit operating in an oscillator should jump frequency from one response to the next. This was actually observed in a Miller-type oscillator at the stated $C_{t}$ value. It should be observed that with this crystal unit the envelopes (or tangents) of the $R_{p}$ and frequency curves at the two resonances have practically normal slopes. It would, therefore, be misleading to draw an $R_{p}-f-C_{t}$ characteristic from a few points. One must be careful not to associate an $f_{r}$ of one response with the $f_{a}$ of another.

It is probable that the presence of an interfering response would not show up in test-oscillatorcircuits now in use. This particular abnormality was detected in other measurements; such as, in improper correlation of $I_{g}$ versus $R_{p}$, operation of an oscillator at a response with a relatively higher $R_{s}$, and an unusually wide $\Delta f$. The frequency spectrum of the crystal is probably the best indicator of possible presence of interfering responses. The spectrum of this particular unplated unit is shown in figure 14 and should be compared with figure 8 . It demonstrates the proximity and the relative values of $R_{p}, R_{s}$, and $\Delta f$ of all responses.

The low-resistance end of the second response was explored carefully. Equivalent $X_{e}$ and $R_{e}$ values of the latter are plotted in figure 15 together with similar curves of normally behaving crystals. Figure 15, a shows $R_{e}$ and $X_{e}$ of the first response of this "complex" crystal and 15- $B$ shows these curves for the second response with a minimum $R_{e}$ at a frequency above $f_{r}$. Figure 15 , c and $\mathrm{d}$ show the same characteristics of the major 


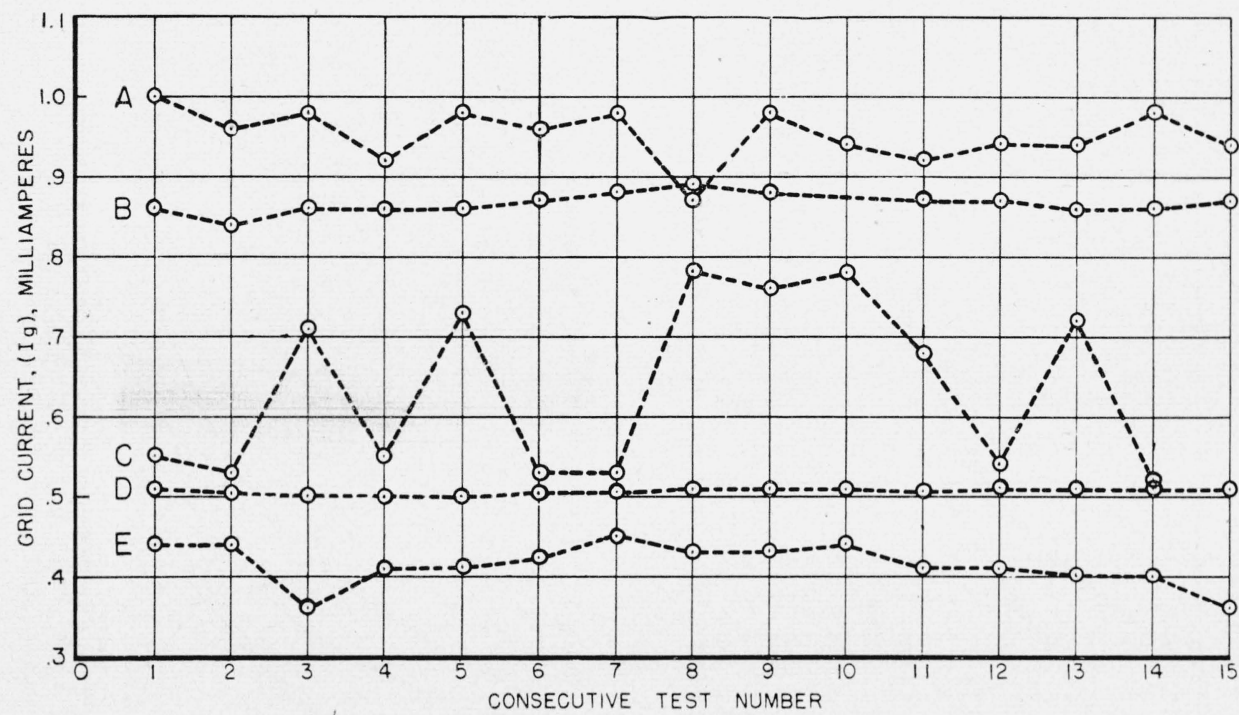

FIGURE 12.-Crystal unit stability in terms of grid-current variations of a Miller-type oscillator.

Consecutive tests were made at approximately 24-hour intervals. $A$, an air-gap crystal unit which represented the stability of the majority of this type of unit; $B$, an air-gap crystal unit which represented the minimum stability required for reliable measurements; $C$, an air-gap crystal unit which was extremely unstable; $D$, a wire-mounted crystal unit with metal-film electrodes. This unit maintained superior stability; $E$, another wire-mounted crystal unit with metal-film electrcdes. This unit was more representative of the stability of the majority of this type of unit.

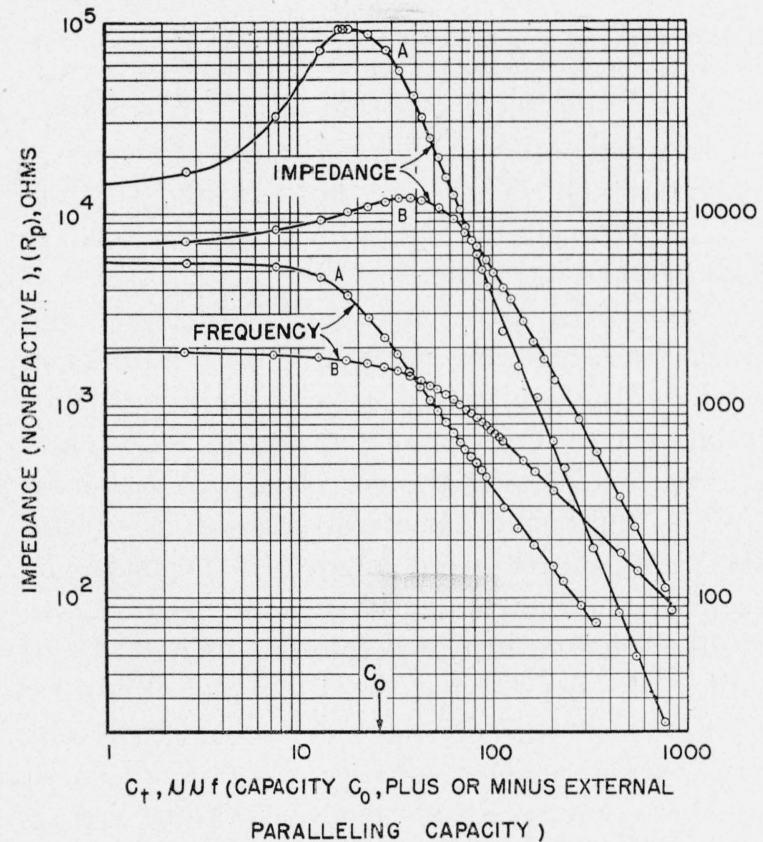

FIGURE 13.-Electrical characteristics of two interfering responses of an air-gap, quartz-crystal unit.

$$
A, f_{r}=8,697.870 \mathrm{kc} / \mathrm{s} ; B, f_{r}=8,700.395 \mathrm{ke} / \mathrm{s} \text {. }
$$

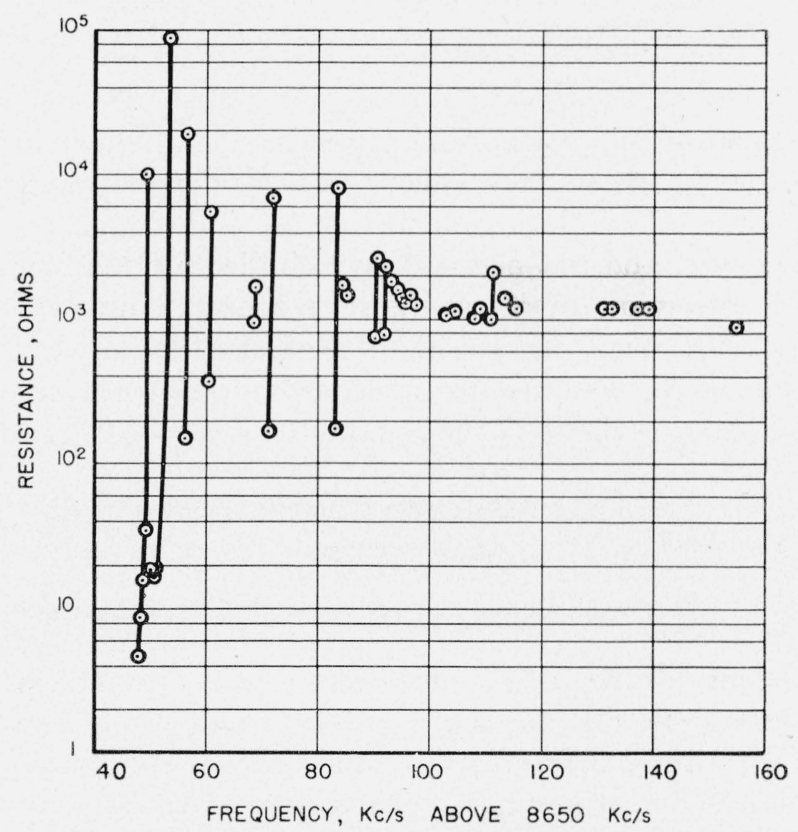

FIgURE 14.-Frequency response spectrum of an air-gap, pressure mounted quariz-crystal unit, typical of those tested.

The large number of responses of this type of crystal unit may be eom pared with figure 8 , which shows those of a wire-mounted crystal having metal-film electrodes. The position and magnitude of antiresonant impedance of the first two responses are indicative of the behavior shown in figure 13 and described in the text. 

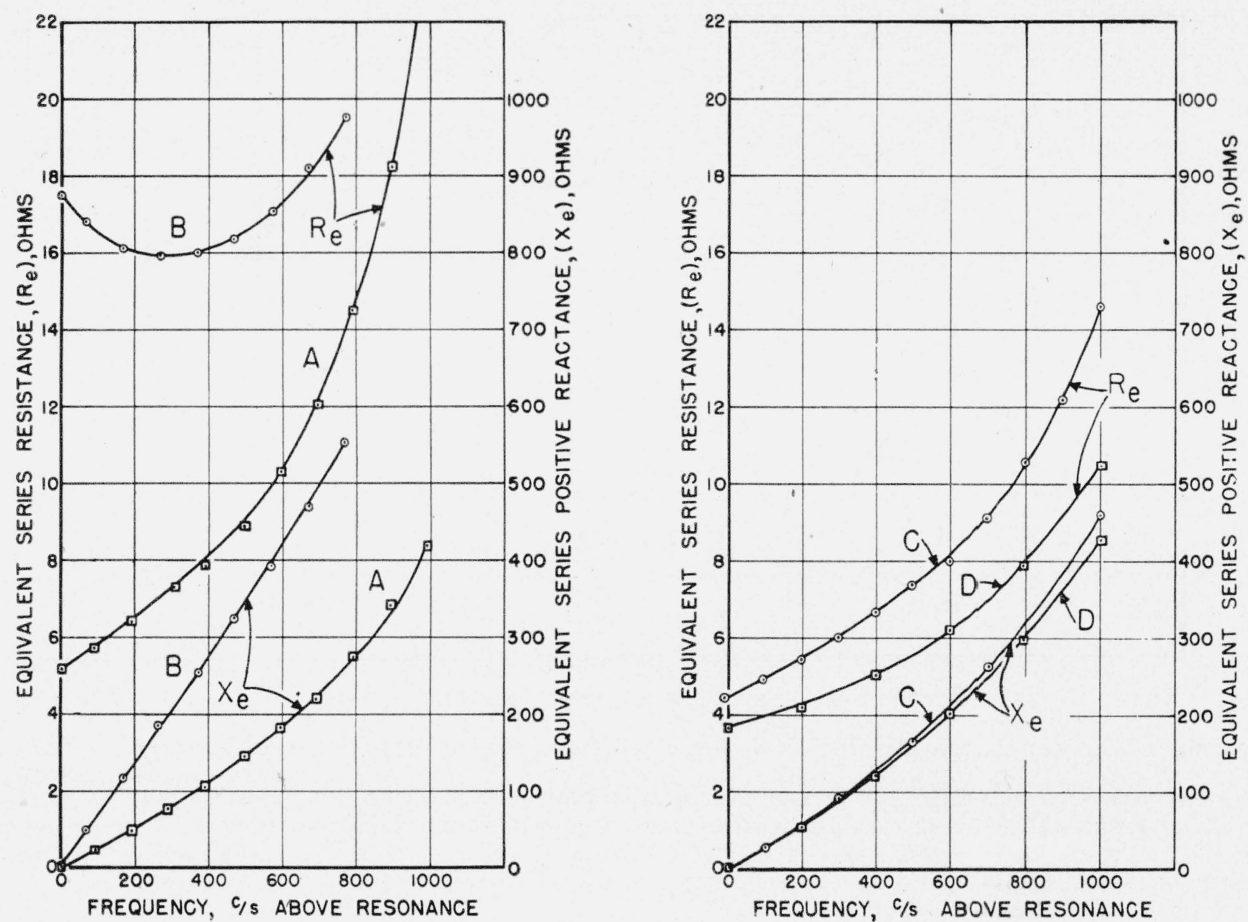

FIGURE 15.-Electrical characterisiics near series resonance of iwo responses of a complex crystal unit (left), and of the major responses of two "normal" crystal units (right).

All crystals were 8.7 Mc, BT-cut, air-gap, pressure mounted. $A$, Major response: complex crystal unit; $B$, second response: complex crystal unit. $C$, major response: normal crystal unit; $D$, second response: normal crystal unit. Other characteristics of the complex unit are shown in figures 13 and 14.

responses of two normal crystals. The object in showing these is to demonstrate the practical means of exploring behavior of individual responses, and not necessarily to indicate a manner of behavior of a complex response. As was pointed out, the study of this type of crystal should be handled as a separate problem, employing methods of measurements described here.

\section{Correlation Between Characteristics and Performance}

(a) Effect of Amplitude of Vibration and of Temperature

One of the major considerations in the field of quartz-crystal applications for some time has been the establishment of an index of performance to correlate the quality of a unit with its activity and stability in an oscillator, and with its effectiveness in wave-filter circuits.

It has been shown in a number of publications $[4,6,9]$ that the activity of an oscillator is a function of the equivalent dynamic resistance $R_{p}$ of the crystal unit. It was mentioned in the introduction that the higher the $Q_{x}$ of a crystal unit, the higher its stability and effectiveness in electric circuits. Therefore, both $R_{p}$ and $Q_{x}$ seem to be the parameters required to state the relative merits of a crystal unit.

To verify the above relationship, activity and $R_{p}$ measurements were performed on a number of crystal units of varying degree of stability. The correlation curves are shown in figures 16,17 , and 18. Figure 16 shows the correlation of $I_{\mathrm{g}}$ versus $R_{p}$ of a number of relatively unstable crystal units. All efforts were made to measure $R_{p}$ immediately after an activity measurement, to prevent a change in characteristics as a result of handling the crystal unit. To further minimize the differences, the activity was reduced by lowering the oscillator plate voltage. $R_{p}$ was measured with the $Q$-meter at low-voltage values. It is seen that there is a fairly good correlation between low and high activity crystals with respective $R_{p}$ values. Moreover, there is a good correlation between variations in activity of individual crystals and respective variations in their $R_{p}$ values. For example, crystal 11 had three values of activity obtained at three different times, and three respec- 


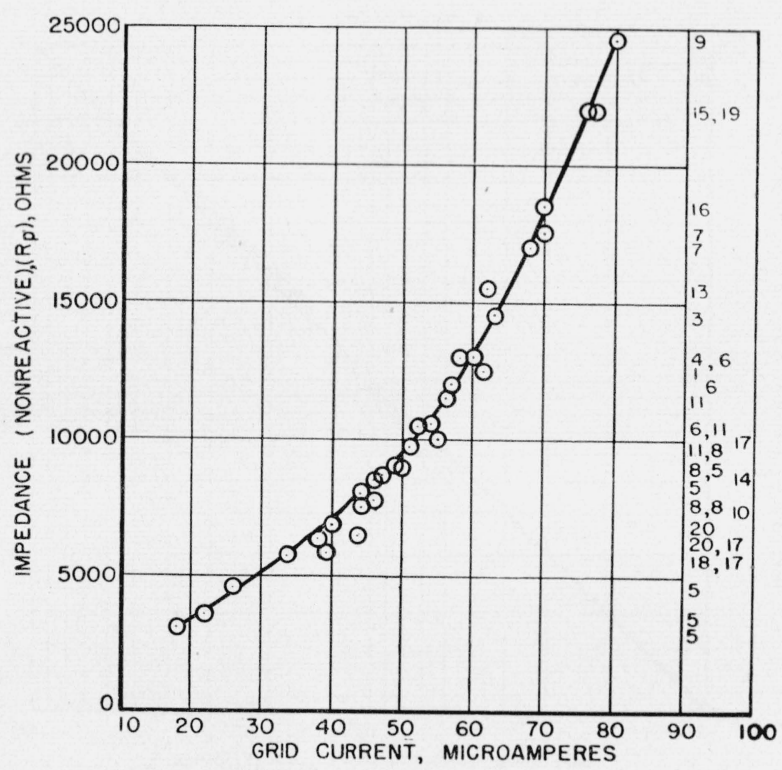

Figure 16.-Correlation between $R_{p}$ and $I_{g}$ of a number of 8.7-Mc crystal units: BT-cut, air-gap type.

Each crystal unit was measured several times. Numbers 9, 15, etc, are examples of stable crystals. Numbers 5 and 8 are examples of poor stability In all measuremnts the $R_{p}-I_{v}$ correlation is apparent.

tive $R_{p}$ values. All seem to fall well within the curve of the $I_{g}-R_{p}$ function for all crystals.

Figure 17 shows this correlation for 13 relatively stable units, with three values of $\Delta C_{t}$ and $R_{p}$ measured at high voltage values (approximately those across the crystal terminals in a Millertype oscillator at normal plate voltage). There seems to be good correlation between $I_{g}$ and $R_{p}$ in all instances.

The correlation between full and reduced activity and $R_{p}$ values of five of the most stable units of the group of figure 17 is shown in figure 18 . Six points were obtained with each unit, three at full and three at reduced activity. Each of the three points corresponds to a $\Delta C_{t}$ value of 25,40 , and $70 \mu \mu f$, respectively, in parallel with $C_{o}$. All the $R_{p}$ values were measured at low voltage values with the $Q$-meter.

The effect of voltage level across the crystal on its characteristics was checked in measuring $R_{s}$ at crystal-current values from a fraction of a milliampere up to 100 milliamperes. No differences in this parameter were observed. Low-level versus high-level $R_{p}$ curves are given in figure 19 for the wire-supported units and in figure 20 for some of the more stable pressure-mounted type. Here again, the evidence indicates that the amplitude

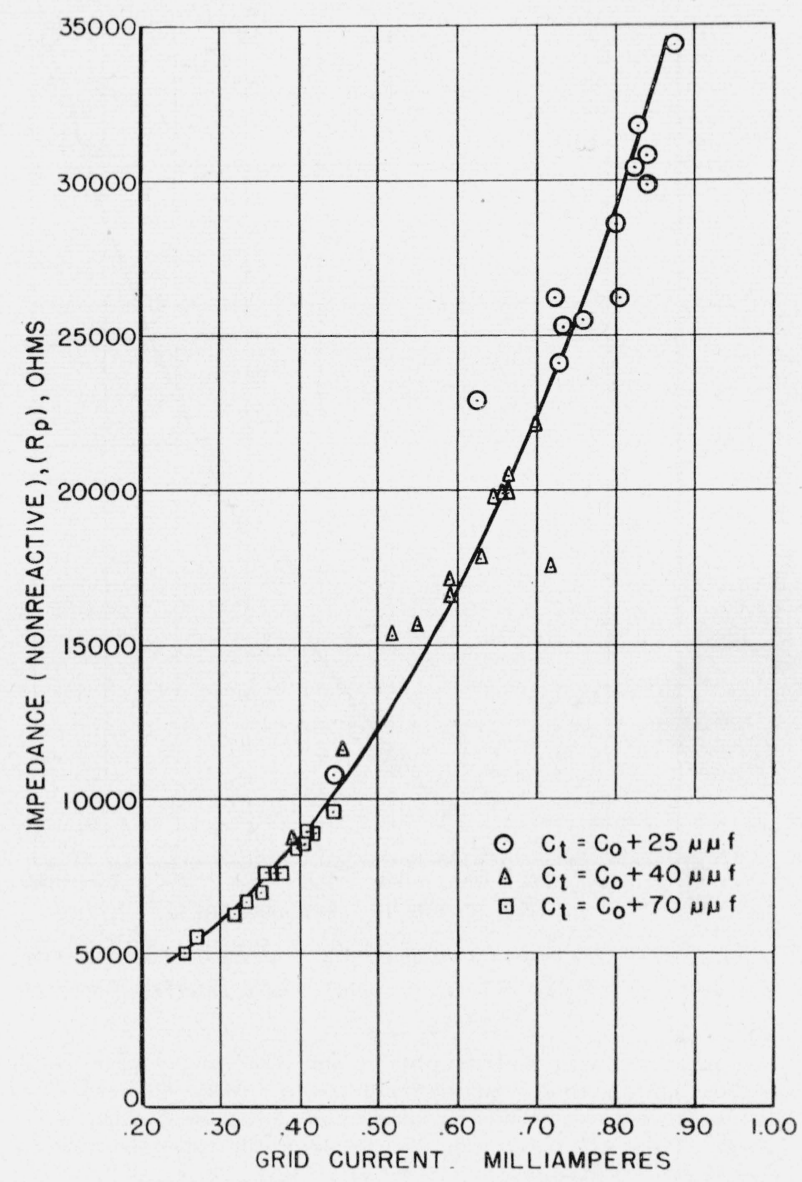

FIGURE 17.-Correlation between $R_{p}$ and $I_{g}$ of thirteen 8.7Mc, air-gap, pressure-mounted crystal units.

of the crystal vibration does not affect its fundamental electrical equivalent characteristics. It is believed that the scattering of some of the points in figure 20 were caused by instability of the crystal units and not by an actual difference between the $R_{p}$ values at the two levels.

Figure 21 shows the temperature effect and correlation between series resistance $\left(R_{s}\right)$ and grid current in a Miller-type oscillator of two wiremounted crystal units. Although for crystal $A$ both $I_{g}$ and $R_{s}$ show some random variations about a fairly constant average value, for crystal $B$ they have definite slopes of opposite sign.

As $I_{g}$ is a function of $R_{p}$ it is also a function of $R_{s}$ and of $C_{t}$ (see eq 2). Observations were therefore made on the effect of temperature on $C_{0}$. No detectable variations were found. It may then be assumed that the $I_{g}$ variations were caused entirely by $R_{s}$ changes. 


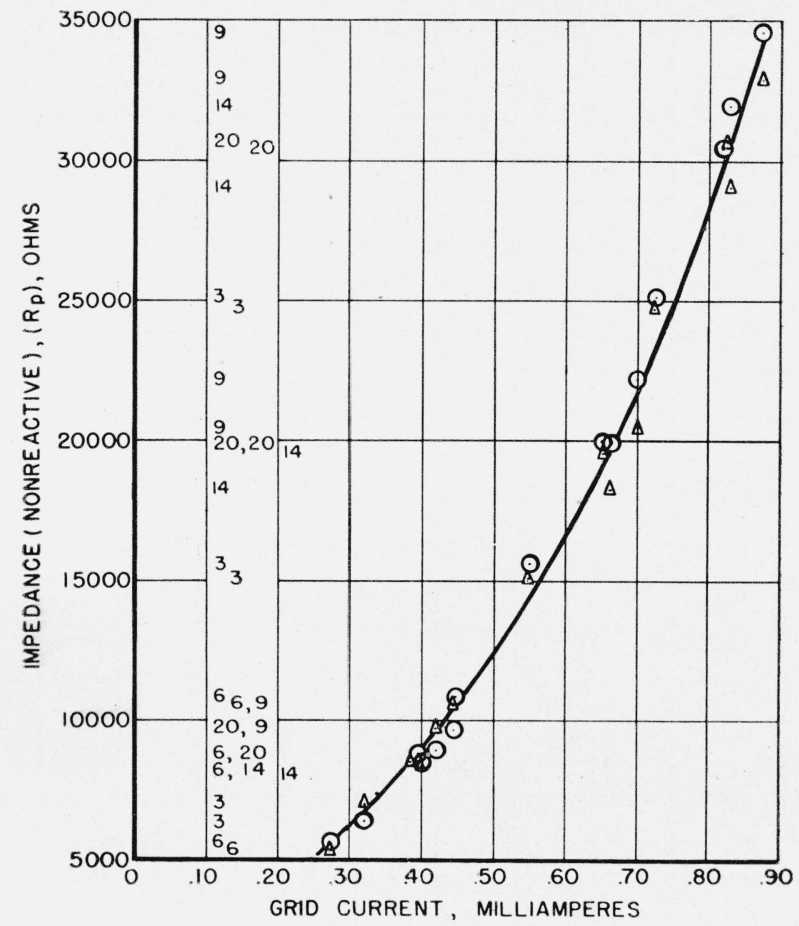

Figure 18.-Correlation between $R_{p}$ and $I_{g}$ at two different $r-f$ voltages of five 8.7-Mc crystal units selected for good stability.

The circles represent measurements at impedance and voltage values corresponding to normal operating conditions in a Miller-type oscillator. The triangles represent values for approximately 10 percent of the above voltages. Three $\Delta C_{t}$ values were used to obtain three corresponding $R_{p}$ values for each crystal unit.

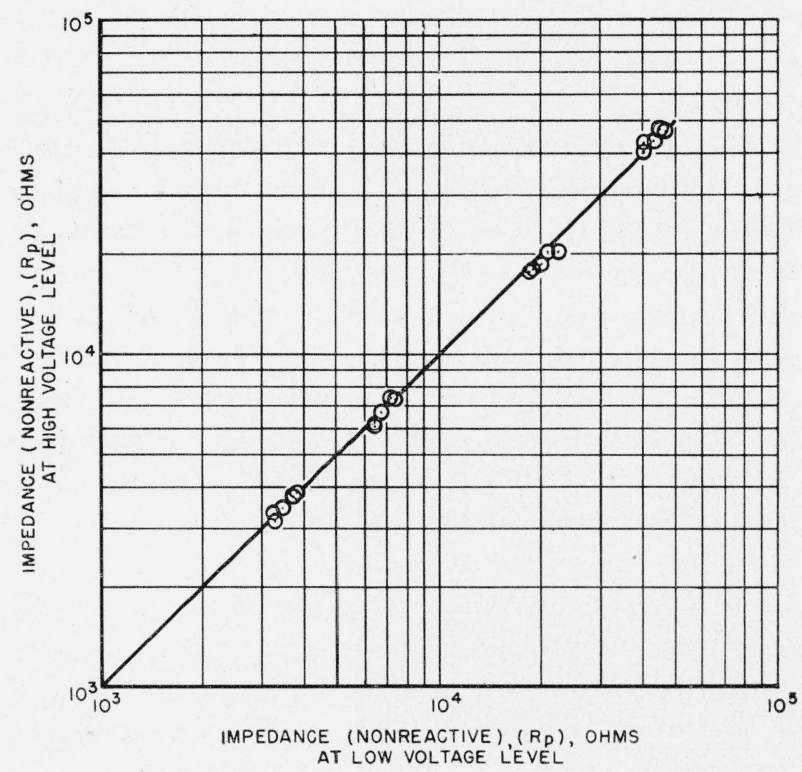

Figure 19.-Correlalion between $R_{p}$ values measured at high and at low $r$-f voltages across the crystal.

Five $8.7-\mathrm{Mc}$ crystal units (metal-film electrodes, wire mounted) were measured by using four different values of shunting capacitance $\left(\Delta C_{t}\right)$.

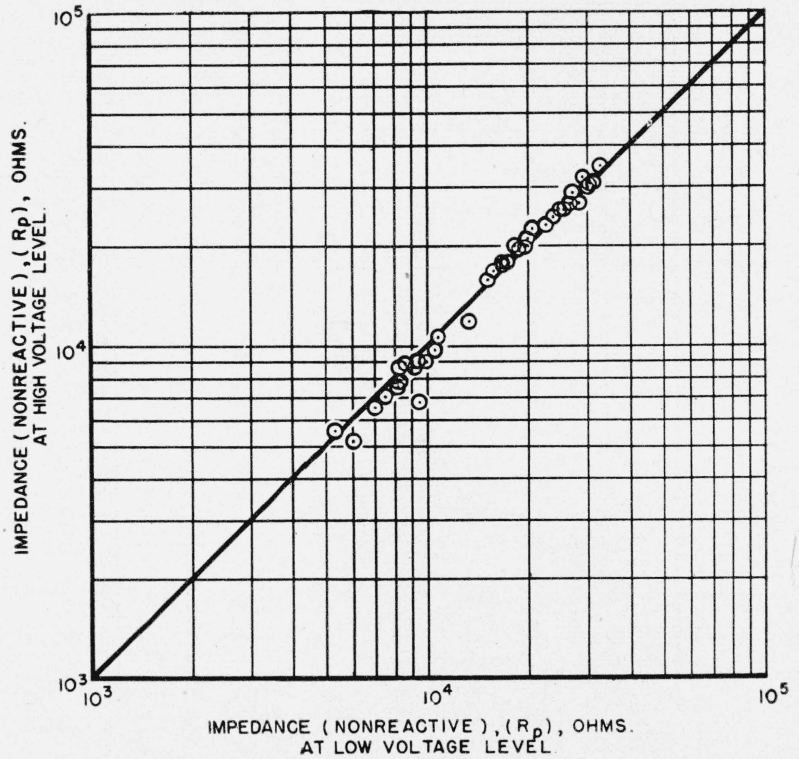

FiguRE 20.- Correlation between $R_{p}$ values measured at high and at low $r-f$ voltages across the crystal.

Five 8.7-Mc crystal-units (air-gap, pressure mounted) were measured by using four different values of shunting capacitance $\left(\Delta C_{t}\right)$.

Variations of $R_{s}$ were measured with the crystal under test placed in a temperature-controlled cabinet and connected to the impedance bridge through a half-wavelength coaxial cable. Corrections were made for losses in the cable. No difficulties were encountered in the application of a coaxial cable to these measurements.

Temperature effects on the relative position of crystal-unit responses and on their $R_{p}, R_{s}$, and $\Delta f$ values were measured as a preliminary step in the study of activity dips. They are shown in figure 22 for a normal wire-mounted crystal unit. Three

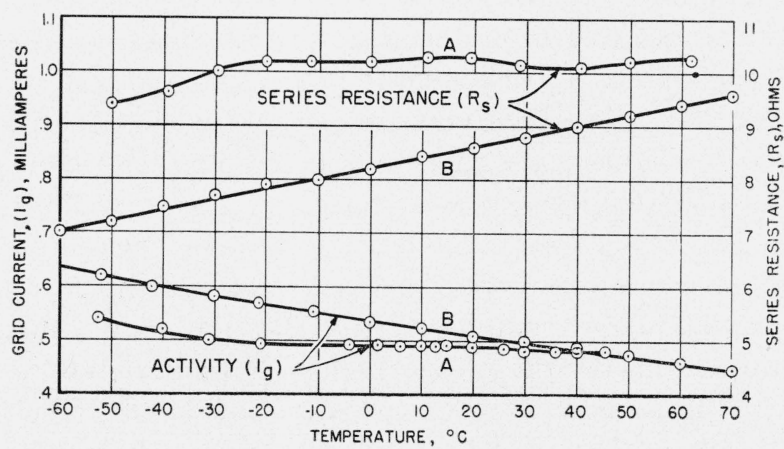

Figure 21.-Variation of the series resistance of crysial units and of the grid current with temperaiure.

8.7-Me crystal unit: $B T$-cut; metal-film electrodes, wire mounted. " $A$ " refers to one unit; " $B$ " to the other. 


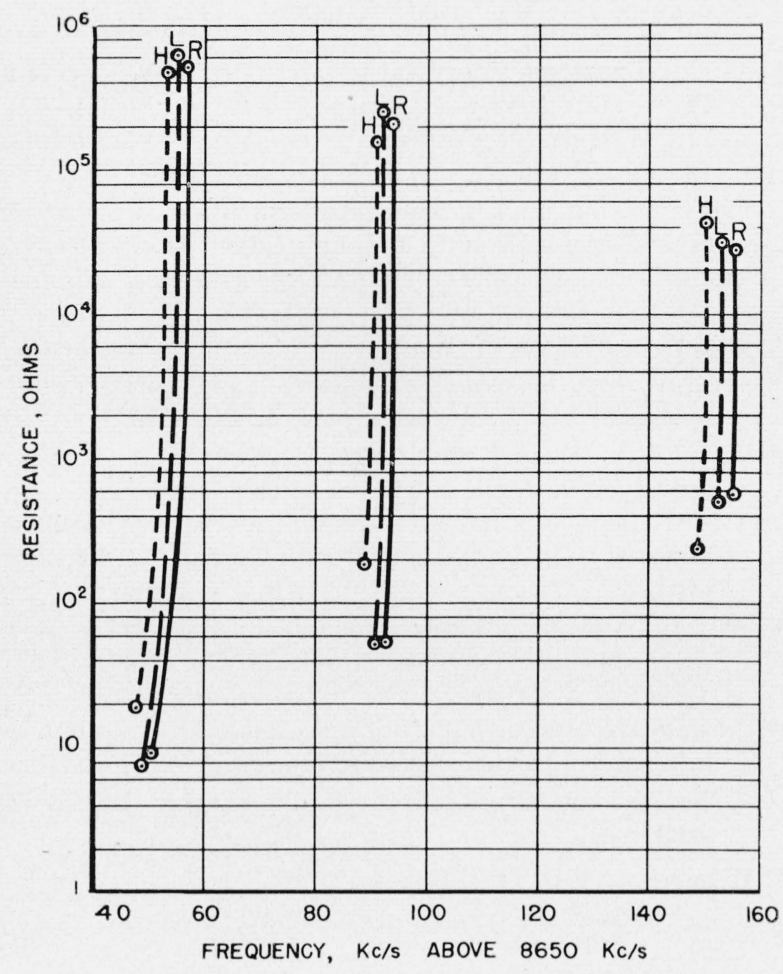

Figure 22.-Effect of temperature on three relatively large, nearby responses of an $8.7-M c$ crystal unit; BT cut, metalfilm electrodes, wire mounted (high, low, and room temperatures).

large responses only are shown merely to demonstrate the effectivenesss of the method. Other responses of normal as well as of complex crystal units were under observation; the results are as yet inconclusive and are not given here.

Another graphical presentation of the effect of temperature on the quartz crystal unit is shown in figure 23 . In curve $A$, a relatively high crystal current resulted in a resonance frequency turning point at an apparent ambient temperature of $3^{\circ} \mathrm{C}$. Actually, the high crystal current brought the enclosed crystal plate to a temperature of about $44^{\circ} \mathrm{C}$ (the "normal" turning point), even though the ambient indicating thermometer read $3^{\circ} \mathrm{C}$. Curve $B$ shows the resultant shift in frequency turning point to an ambient temperature of $15^{\circ} \mathrm{C}$ as the crystal assembly was removed from its enclosure and was thereby exposed to free ventilation. In curve $C$, the crystal current was made

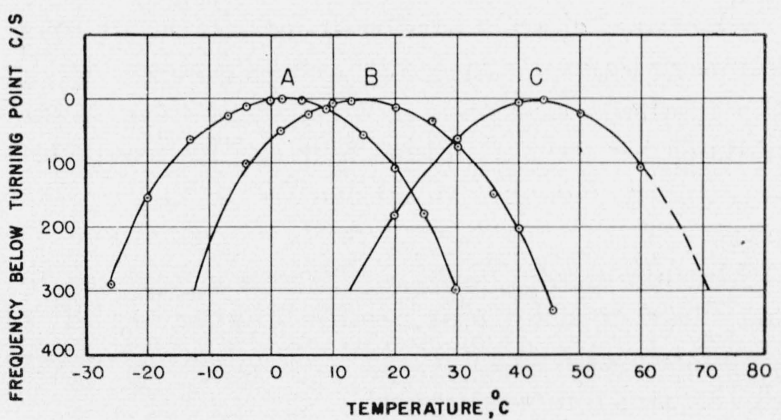

Figure 23.-Effect of operating conditions on temperature and frequency turning position at resonance of an 8.7-Mc crystal; BT cut, metal-film electrodes, wire mounted.

$A$, Crystal in plastic container, measured with relatively high crystal current; $B$, same as $A$, with crystal removed from container; $C$, crystal in plastic container, measured at resonance with relatively low crystal current.

negligible and a full $44^{\circ} \mathrm{C}$ ambient temperature was necessary to bring the crystal plate to its frequency turning-point temperature.

Thus any predetermination of frequency turning point with temperature by a manufacturer or a design engineer must take into consideration at least the approximate amplitude of oscillation expected of the quartz crystal unit. This is in keeping with the observation that the performance index and $\Delta f$ versus $C_{t}$ curves used in design work should be accompanied by temperature data for a more complete expression of the characteristics of a quartz-crystal unit.

\section{Conclusion}

It was shown that comprehensive data on electrical characteristics of quartz-crystal units may be obtained with $\mathrm{r}-\mathrm{f}$ bridges and $\mathrm{Q}$-meters and a source of adjustable stable c-w frequency. Impedance above and below the maximum antiresonance frequency, up to $5,000,000 \mathrm{ohms}$, may be measured to \pm 5 percent or better.

Graphical presentation of measured characteristics as straight lines is a form believed to be particularly suitable for design purposes as they supply essential electrical characteristics.

Results indicated that a good crystal unit was practically unaffected by the normally used voltages across the crystal unit, i. e., no changes with amplitude were observed on units whose series resistance was constant with temperature. 
Electrical characteristics of interfering or overlapping responses may be precisely measured by the methods described. Measuring circuits employing the crystal under test as the frequencycontrolling element could hardly serve the purpose.

Measurements based on frequency presetting may lead to erroneous results because of shift of responses with crystal currents, affecting in turn the crystal temperature.

The greatest difficulty in measuring electrical characteristics and establishing figures of merit of quartz-crystal units seemed to be the inability to reproduce results as a consequence of changes in the unit. This difficulty could apparently be entirely eliminated by superior types of mounting and construction. The crystal unit would then have good possibilities as reference standards of impedance as well as frequency.

The investigations described were of great value in connection with the precision calibration of new types of crystal unit test sets.

\section{References}

[1] G. M. Thurston, A crystal test set, Bell Labs. Record 22, No. 12 (1944).

[2] W. E. McNatt, Test set for quartz crystals, Electronics 18, No. 4 (1945).

[3] Karl S. Van Dyke, The standardization of quartzcrystal units, Proc. Inst. Radio Engrs. 33, 15 (1945).

[4] R. A. Heising, Quartz crystals for electrical circuits (D. Van Nostrand Co., Inc., New York, N. Y., 1946).

[5] Geoffrey Builder, A note on the determination of the equivalent electrical constants of a quartz-crystal resonator, A. W. A. Tech. Rev. 5, 41 (1940).

[6] I. E. Fair, Piezoelectric crystals in oscillator circuits, Bell System Tech. J. 24, 161 (1945).

[7] C. W. Harrison, The measurement of the performance index of quartz plates, Bell System Tech. J. 24, 217 (1945).

[8] W. P. Mason, Electro-mechanical transducers and wave filters, (D. Van Nostrand Co., Inc., New York, N. Y., 1942).

[9] M. Boella, Performance of piezo-oscillators and the influence of the decrement of quartz on the frequency oscillations. Proc. Inst. Radio Engrs. 19, 1252 (1931).

Washington, October 4, 1946. 